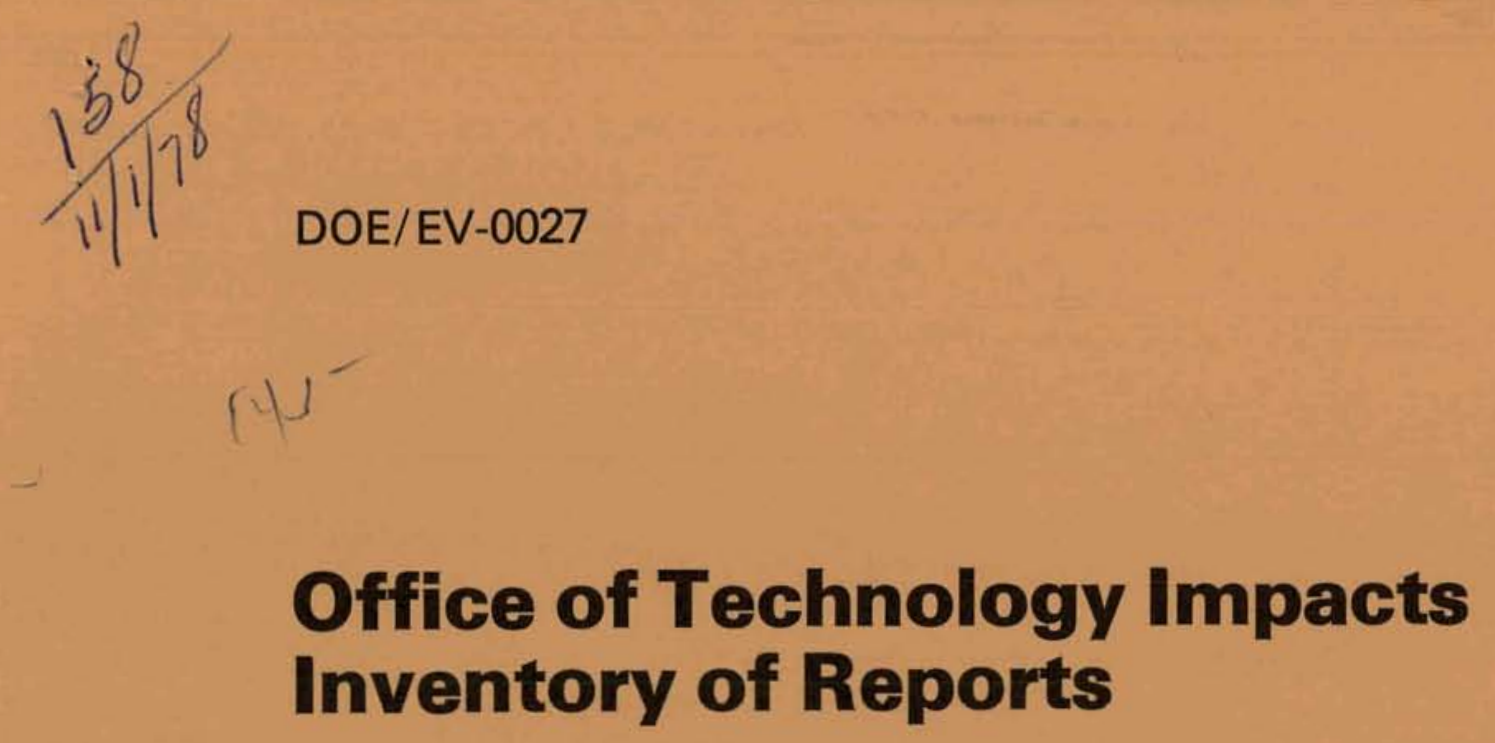

DOE/EV-0027

\title{
Office of Technology Impacts Inventory of Reports
}

October 1978

U.S. Department of Energy

Assistant Secretary for Environment Office of Technology Impacts

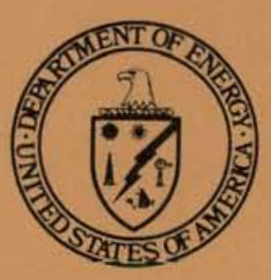




\section{DISCLAIMER}

This report was prepared as an account of work sponsored by an agency of the United States Government. Neither the United States Government nor any agency Thereof, nor any of their employees, makes any warranty, express or implied, or assumes any legal liability or responsibility for the accuracy, completeness, or usefulness of any information, apparatus, product, or process disclosed, or represents that its use would not infringe privately owned rights. Reference herein to any specific commercial product, process, or service by trade name, trademark, manufacturer, or otherwise does not necessarily constitute or imply its endorsement, recommendation, or favoring by the United States Government or any agency thereof. The views and opinions of authors expressed herein do not necessarily state or reflect those of the United States Government or any agency thereof. 


\section{DISCLAIMER}

Portions of this document may be illegible in electronic image products. Images are produced from the best available original document. 
Available from:

National Technical Information Service (NTIS) U.S. Department of Commerce

5285 Port Royal Road

Springfield, Virginia 22161

Price: Printed copy: $\$ 4.50$

Microfiche: $\quad \$ 3.00$ 
DOE/EV-0027

Dist. Category UC-13

\section{Office of Technology Impacts Inventory of Reports}

October 1978

\section{U.S. Department of Energy Assistant Secretary for Environment Office of Technology Impacts \\ Washington, D.C. 20545}

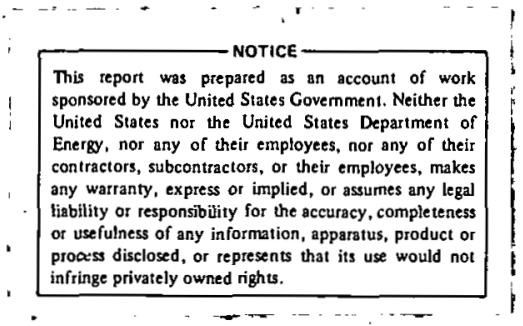


AB STRACT

The Inventory of OTI Reports is a bibliographic listing of reports on environmental analyses, assessments, and related studies sponsored by the office of Technology Impacts (OTI). The Office supports the Assistant Secretary for Environment (ASEV) in the U.S. Department of Energy (DOE). OTI conducts as sessments of the environmental impacts of advanced energy technologies and evaluates policy options and their implications. These analyses are an integral part of ASEV's contribution to DOE's energy policy formulation. Revisions of this Inventory are planned on an annual basis. 
OFFICE OF TECHNOLOGY IMPACTS

Inventory of Reports

Page No.

ABSTRACT

Introduction

Summary of Annual Reports

Major Reports Released and Topics Addressed

Summary of High Priority Reports

Inventory of Reports by Subject Areas

I. Policy Analysis

II. Technology Assessment

A. Coal

B. Geothermal Energy

C. Environmental Development Plans (EDPs)

III. National Impact Assessment

A. NEP

B.. Water for Energy

C. Special Studies

IV. Regional Impacts Assessment

A. Northeast

B. Midwest

C. Southeast

D. Rocky Mountain

E. Far West

F. Pacific Northwest

G. Offsite 
INTRODUCTION

This Inventory of OTI Reports is a bibliographic listing of reports sponsored by the office of Technology Impacts (OTI). This listing contains reports produced as of April 1978, by OTI and its predecessor offices under the former Energy Research and Development Administration (ERDA). To provide others with information on the products and progress of OTI assessment and policy evaluation efforts, the Inventory of OTI Reports will be revised on an annual basis.

In January 1978, OTI was created to analyze the impacts of energy-related environmental policies, laws, and regulations, to assess the environmental impacts, identify the environmental issues, and specify the research requirements for energy technologies being developed by DOE, and to 'assess the national and regional environmental, health, and safety impacts of DOE policies and programs. The work of OTI is carried out through four program areas. The functions of each program area are briefly described below.

\section{Policy Analysis Program}

o identifies emerging and existing environmental policy issues,

- conducts options analyses for identifying the environmental impacts of alternative energy policies, and

o evaluates the environmental impacts of alternative energy policies

\section{Technology Assessment Program}

- conducts assessments on the nationwide environmental impacts of specific technologies, and

- prepares and updates EDPs and Environmental Readineśs-Documents:(ERDs)

\section{National Impact Program}

- develops new analytical techniques or adapts existing analytical techniques for assessment needs,

- provides information bases on the energy and environmental research programs, and

- maintains data bases on national economics trends, technology characterization, and other data which are fundamental to the assessment pröcess 


\section{Regional Impacts Program}

- conducts Regional Characterization studies of each of six major regions of the Nation,

- supports an Issue Identification program to define the major environmental issues in each region of the Nation, and

- sponsors special Mitigation Studies and Case Studies which suggest avenues for alleviating or resolving major regional environmental issues

Reports in this Inventory are organized by program area. This Inventory was compiled by Art Garcia and Sandra Rogowski. 
SUMMARY

The current Inventory lists reports prepared as of April 11, 1978, which are under OTI cognizance. As indicated, some of these reports are in draft. form and are pending completion. Of the reports listed, the major completed products of each OTI program area are described below and outlined in Table 1.

1. Policy Analysis.

Evaluation of key state and national legislative precedents for their impact on future energy growth. in all regions of the U.S.

2. Technology Assessment

2.1 The National Coal Utilization Assessment (NCUA) Project for several regions of the Nation.

2.2 An Assessment series on the Imperial Valley Environmental Plan (IVEP).

2.3 Of the 35 EDPs prepared for FY 1977, 11 were completed and released in $f$ inal form.

3. National Impact

3.1 An Environmental Analysis of the (1977) National Energy Plan (NEP) resulting in 8 technology impact reports which investigated the broad national impacts of the NEP.

3.2 Release of the first draft Annual Environmental Analysis Report (AEAR). The AEAR is a detailed analytical investigation of the national environmental impacts of future energy growth to the year 2000 based on the initiatives of the NEP.

3.3 Series on "Water for Energy" in conjunction with the Water Resources Council (WRC).

3.4 The "FY 1976 Federal Inventory."

4. Regional Impacts

4.1 Perspectives on the Energy Future of the Northeast.

4.2 Series on "Environmental Characterization of the Lake Michigan Region." 
4.3 Series on "Socioeconomic Assessment of Energy Development in Mercer County, North Dakota."

4.4 Series on "Air Quality and Energy Development in the Rocky Mountain West."

4.5 Series on "Energy Development in Resource Rich States." 
TABLE 1. OUTLINE OF MAJOR OTI PROJECTS AS OF APRIL 11,1978

\section{MAJOR OTI PROJECTS}

1. Policy Analysis -

State and National

Legislative Precedents

2. Technology Assessment

2.1 NCUA

\subsection{IVEP}

2.3 EDPs (finalized reports only)

3. National Impact

3.1 NEP Analyses

\section{REPORT INDEX NUMBERS}

BNL-MT-5
LLL-MT-3
LLL-CC-1
OF F-MT-4

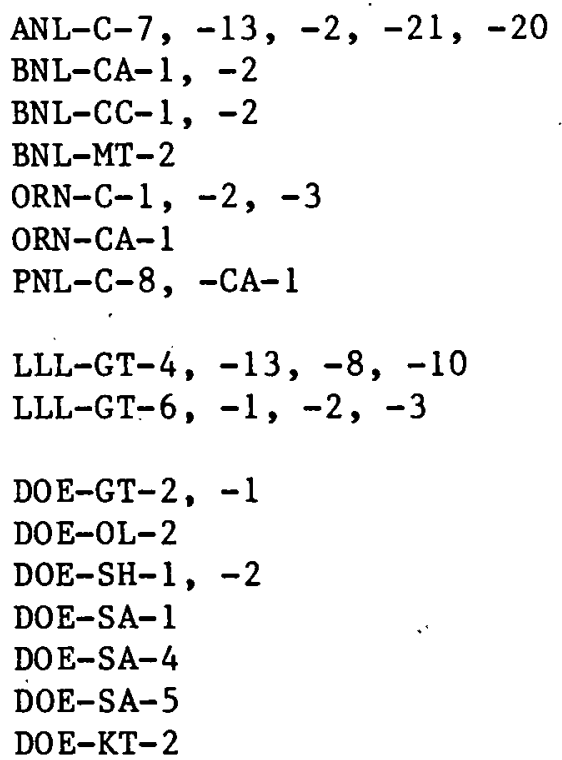

ANL-MT-6

ANL-C- 10

BNL-CC-3

BNL-MT-3

LAS-SA-1

ORN-MT-17

$O F F-C-1,-2$

\section{APPLICABLE REGIONS OR TECHNOLOGIES}

Northeast
California
California
U.S.A.

U.S.A.

Midwest

Northeast

Northeast

Northeast

Southwest

Southwest

Nor thwest

California

California

Geothermal

0il Shale

Solar Heating

Ocean Thermal

Solar Thermal Power

Photovoltaics

Electric Vehicle

U.S.A

U.S.A.

U.S.A.

Northeast

U.S.A.

U.S.A.

U.S.A. 


\section{MAJOR OTI PROJECTS}

3.2 AEAR - 1977

3.3 Water for Energy

\subsection{Federal Inventory -.1976 \\ 4. Regional Impacts \\ 4.1 Perspectives $"$ Northeast \\ 4.2 Environmental Characterization of Lake Michigan Region \\ 4.3 Energy Development in Mercer County, North Dakota \\ 4.4 Air Quality in the Rocky Mountain West \\ 4.5 Energy Development in Resource Rich States}

\section{REPORT INDEX NUMBERS}

OFF-MT- $37,-38,-1$

BNL-MT-19

LAS-MT-15, -16

ORN-MT-44, -45

OFF- $27,-28,-31,-32$

OFF-35

OFE -36

OFF-MT- 5

BNL-MT- 12

ANL-MT-5, $-12,-13$

ANL-MT- $29,-10,-14$

ANL-MT-20, $-21,-22$

ANL-MT-23, $-24,-16$

$\mathrm{ANL}-\mathrm{CA}-7,-6$

LAS-MT- $2,-3,-4$

OFF-MT-9

OF F-MT- 10

OFF-MT- 11

OFF-MT-12

OFF-MT-3

\section{APPLICABLE REGIONS OR TECHNOLOGIES}

U.S.A.

U.S.A.

Western U.S.A.

Eastern U.S.A.

U.S.A.

Appalachia

River Basins

U.S.A.

Northeast

Lake Michigan

Lake Michigan

Lake Michigan

Lake Michigan

North Dakota.

Röky Mountain

Texas

North Dakota

Wyoming

Colorado

Alaska 
OFFICE OF TECHNOLOGY IMPACTS INVENTORY OF REPORTS

SUBJECT AREA: POLICY ANALYSIS

\begin{tabular}{|c|c|c|c|c|c|}
\hline $\begin{array}{r}\text { OTI INDEX } \\
\text { NUMBER } \\
\end{array}$ & TITLE & $\begin{array}{c}\text { ON } \\
\text { NTIS } \\
\end{array}$ & AUTHOR (S) & $\begin{array}{l}\text { DATE/ } \\
\text { STATUS }\end{array}$ & $\begin{array}{l}\text { REGION/ } \\
\text { REPORT NUMBER }\end{array}$ \\
\hline OFF-MT- 18 & $\begin{array}{l}\text { Social Science Energy Review Vol. } 1 \\
\text { No. } 1\end{array}$ & No & Yale U. & $\begin{array}{l}\text { Winter } \\
78 \\
\text { Final }\end{array}$ & $\begin{array}{l}\text { U. S. A. } \\
\mathrm{COO}-4287-7 \\
\because \therefore\end{array}$ \\
\hline OFF-MT-13 & $\begin{array}{l}\text { "No't on my Block You Don't : Facility } \\
\text { Siting and the Strategic Importance of } \\
\text { Compensation }\end{array}$ & No & $\begin{array}{l}\text { M. O' Hare } \\
\text { MIT : }\end{array}$ & $\begin{array}{l}\text { Fa11 } \\
1977 \\
\text { Final }\end{array}$ & $\begin{array}{cc}\text { U. } & \text { S. } \\
\vdots & \text { A. } \\
\vdots & \vdots\end{array}$ \\
\hline OFF-MT-4 & $\begin{array}{l}\text { Impact of The Safe Drinking Water Act } \\
\text { on Energy Development }\end{array}$ & No & $\begin{array}{l}\text { Energy } \\
\text { Resources } \\
\text { Co., Inc. }\end{array}$ & $\begin{array}{l}9 / 77 \\
\text { Draft }\end{array}$ & U. S. A. \\
\hline OFF-MT-14 & $\begin{array}{l}\text { Managing the Socioeconomic Impacts of } \\
\text { Energy Development }\end{array}$ & No & OPA\&E & $\begin{array}{l}9 / 77 \\
\text { Final }\end{array}$ & $\begin{array}{l}\text { U. S. A. } \\
\text { ERDA } 77-79\end{array}$ \\
\hline OF F-MT- 15 & $\begin{array}{l}\text { Models and Methodologies for Assessing } \\
\text { the Impact of Energy Developments }\end{array}$ & No & OPA\&E & $\begin{array}{l}9 / 77 \\
\text { Final }\end{array}$ & $\begin{array}{l}\text { U. S. } \ddot{A} \cdot \\
\text { ERDA } 77-91\end{array}$ \\
\hline OF F-MT- 16 & Assistance from Energy Developers & No & OPA\&E & $\begin{array}{l}9 / 77 \\
\text { Final }\end{array}$ & $\begin{array}{l}\text { U. S. A. } \\
\text { ERDA } 77-90\end{array}$ \\
\hline OF F-MT- 17 & $\begin{array}{l}\text { Opportunities for Company-Community } \\
\text { Cooperation in Mitigating Energy } \\
\text { Facility Impacts }\end{array}$ & No & MIT & $\begin{array}{l}8 / 77 \\
\text { Draft }\end{array}$ & U. S. A. \\
\hline BNL-MT-5 & $\begin{array}{l}\text { State Legislatures and Energy Policy in } \\
\text { the Northeast: Energy Facility Siting } \\
\text { and Legislative Action } \\
\qquad \because \\
\because\end{array}$ & Yes & $\begin{array}{l}\text { D. More } 11 \text {, } \\
\text { G.'Singer }\end{array}$ & $\begin{array}{l}6 / 77 \\
\text { Final }\end{array}$ & $\begin{array}{l}\text { NE } \\
\text { BNL-50679 }\end{array}$ \\
\hline OFF-MT-24 & $\begin{array}{c}\text { Federal Aid to Energy Impacted } \\
\text { Communities - A Review of Related } \\
\text { Programs and Legislative Proposals } \\
\begin{array}{c}\therefore \\
\therefore\end{array} \quad \therefore \quad \\
\therefore\end{array}$ & No & $\begin{array}{l}\text { S. Brody } \\
\text { MIT }\end{array}$ & $\begin{array}{l}5 / 77 \\
\text { Final }\end{array}$ & $\begin{array}{cc}\text { U. S. A. } \\
\\
\cdots \\
& \vdots \\
& \vdots \\
& \\
& \ddots \\
\cdots & \vdots\end{array}$ \\
\hline
\end{tabular}

AS OF: APRIL 11, 1978 
SUBJECT AREA: POLICY ANALYSIS

OTI INDEX NUMBER

LLL-MT-3 Legislative History of the California Environmental Quality Act

LLL-CC-1 The Clean Air Act: $\mathrm{SO}_{2}$ Regulation and Power Plant Operation
AS OF: APRIL 11, 1978

ON DATE/ REGION/ NTIS AUTHOR(S) STATUS REPORT NUMBER

Yes J. Wharton, $12 / 76$

M. Lewis

California

UCRL-52188

Yes

J. Wharton $11 / 76$

Final

California UCRL-52182. 
$\therefore \quad \frac{\text { OFFICE OF TECHNOLOGY IMPACTS }}{\text { INVENTORY OF REPORTS }}$

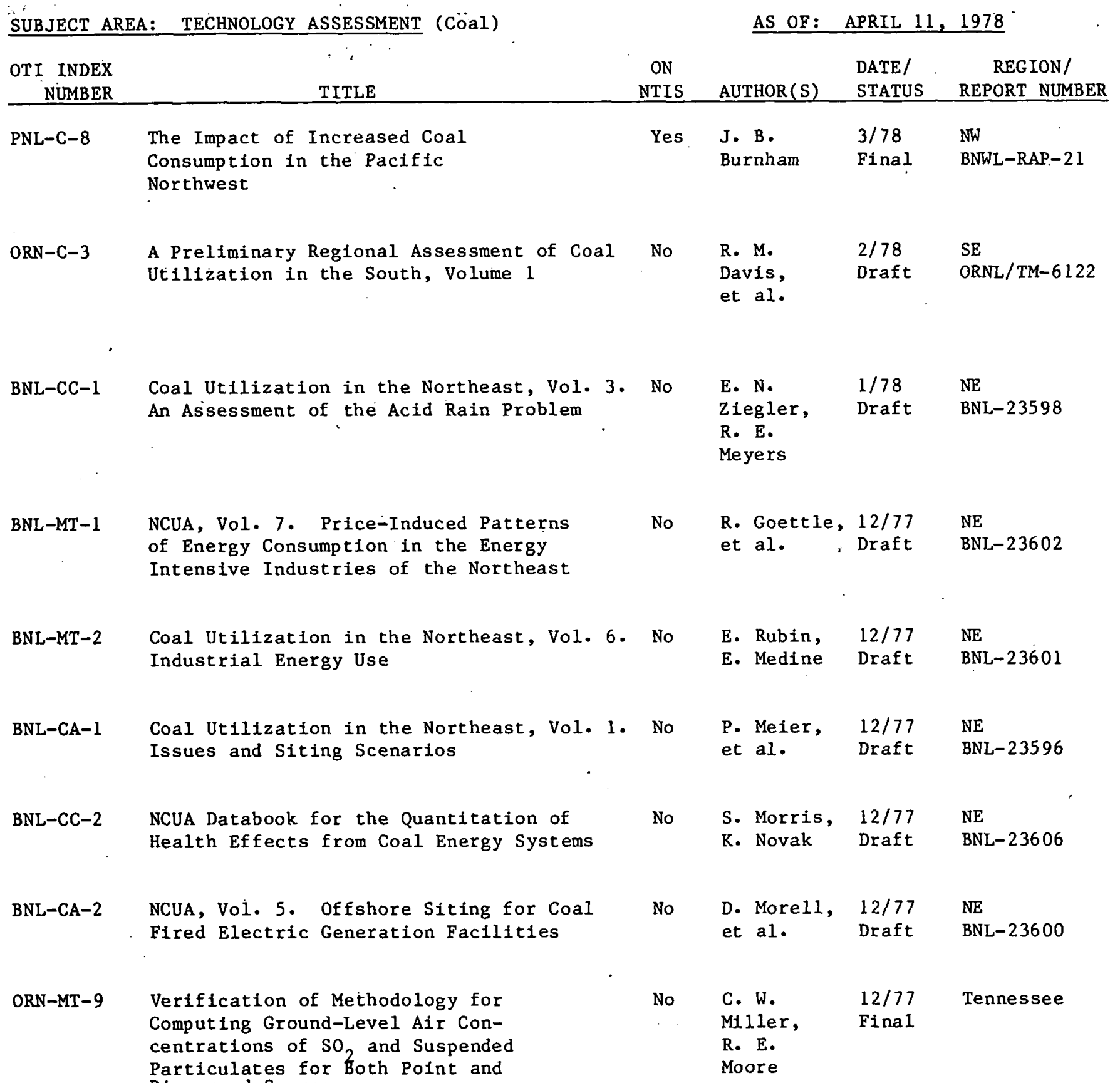




\begin{tabular}{|c|c|c|c|c|c|}
\hline $\begin{array}{r}\text { OTI INDEX } \\
\text { NUMBER } \\
\end{array}$ & TITLE & $\begin{array}{c}\text { ON } \\
\text { NTIS }\end{array}$ & AUTHOR(S) & $\begin{array}{l}\text { DATE/ } \\
\text { STATUS }\end{array}$ & $\begin{array}{c}\text { REGION/ } \\
\text { REPORT NUMBER } \\
\end{array}$ \\
\hline ANL-C-2 & $\begin{array}{l}\text { Coal-Related Issues Identified by State } \\
\text { and Regional Groups in the MIdwest }\end{array}$ & No & $\begin{array}{l}\text { M. L. } \\
\text { Hiltoon }\end{array}$ & $\begin{array}{l}11 / 77 \\
\text { Draft }\end{array}$ & MW \\
\hline ANL $-C-7$ & $\begin{array}{l}\text { National Coal Utilization Assessment } \\
\text { Vol. 1. An Integrated Assessment of } \\
\text { Increased Coal Use in the Midwest: } \\
\text { Impacts and Constraints }\end{array}$ & No & $\begin{array}{l}\text { L. J. } \\
\text { Hoover, } \\
\text { et al. }\end{array}$ & $\begin{array}{l}10 / 77 \\
\text { Draft }\end{array}$ & ANL/A-11 \\
\hline ANL-C- -21 & $\begin{array}{l}\text { National Coal Utilization Assessment. } \\
\text { An Integrated Assessment of Increased } \\
\text { Coal Use In the Midwest: Impacts and } \\
\text { Constraints. Vol. } 2 \text {. }\end{array}$ & No & $\begin{array}{l}\text { L. J. } \\
\text { Hoover; } \\
\text { et al. }\end{array}$ & $\begin{array}{l}10 / 77^{\circ} \\
\text { Draft }\end{array}$ & MN \\
\hline PNL-C-1 & $\begin{array}{l}\text { Constraints on Coal Availability: } \\
\text { The Effects of Depletion }\end{array}$ & No & $\begin{array}{l}\text { T. J. } \\
\text { Foley, } \\
\text { A. E. } \\
\text { Davis }\end{array}$ & $\begin{array}{l}10 / 77 \\
\text { Final }\end{array}$ & Generic \\
\hline ORN-C-1 & $\begin{array}{l}\text { Coal and Energy: A Southern } \\
\text { Perspective -- Regional Characterization } \\
\text { Report for the National Coal Utilization } \\
\text { Assessment }\end{array}$ & Yes & $\begin{array}{l}\text { F. D. } \\
\text { Boercker, } \\
\text { et al. }\end{array}$ & $\begin{array}{l}8 / 77 \\
\text { Final }\end{array}$ & $\begin{array}{l}\text { SE } \\
\text { ORNL/TM-5900 }\end{array}$ \\
\hline ORN $-C A-1$ & $\begin{array}{l}\text { Engineering Evaluations of Coal } \\
\text { Conversion Processes: Part } 1 . \\
\text { Hydrocarbonization and Synthoil- } \\
\text { Information Base, Process Selection, } \\
\text { and Preliminary Evaluation of Alterna- } \\
\text { tives }\end{array}$ & Yes & $\begin{array}{l}\text { R. Salmon, } \\
\text { et al. }\end{array}$ & $\begin{array}{l}8 / 77 \\
\text { Finai }\end{array}$ & $\begin{array}{l}\text { U. S. A. } \\
\text { ORNL/TM-5034 }\end{array}$ \\
\hline ANL-C-20 & $\begin{array}{l}\text { NCUA: The Price and Availability of } \\
\text { Low Sulfur Coal in Eastern Markets } \\
\text { : }\end{array}$ & Yes & $\begin{array}{l}\text { G. C. } \\
\text { Krohm; } \\
\text { C. D. Dux }\end{array}$ & $\begin{array}{l}7 / 77 \\
\text { Final }\end{array}$ & $\mathrm{E}$ \\
\hline ANL-CA-1 & $\begin{array}{l}\text { Preliminary Assessment of the Health } \\
\text { and Environmental Impacts of Fluidized- } \\
\text { Bed Combustion of Coal as Applied to } \\
\text { Electrical Utility Systems }\end{array}$ & No & $\begin{array}{l}\text { K. Hub, } \\
\text { et al. }\end{array}$ & $\begin{array}{l}2 / 77 \\
\text { Final } \\
:\end{array}$ & U. S. A. \\
\hline
\end{tabular}


SUBJECT AREA: TECHNOLOGY ASSESSMENT (Coal)

AS OF: APRIL 11, 1978

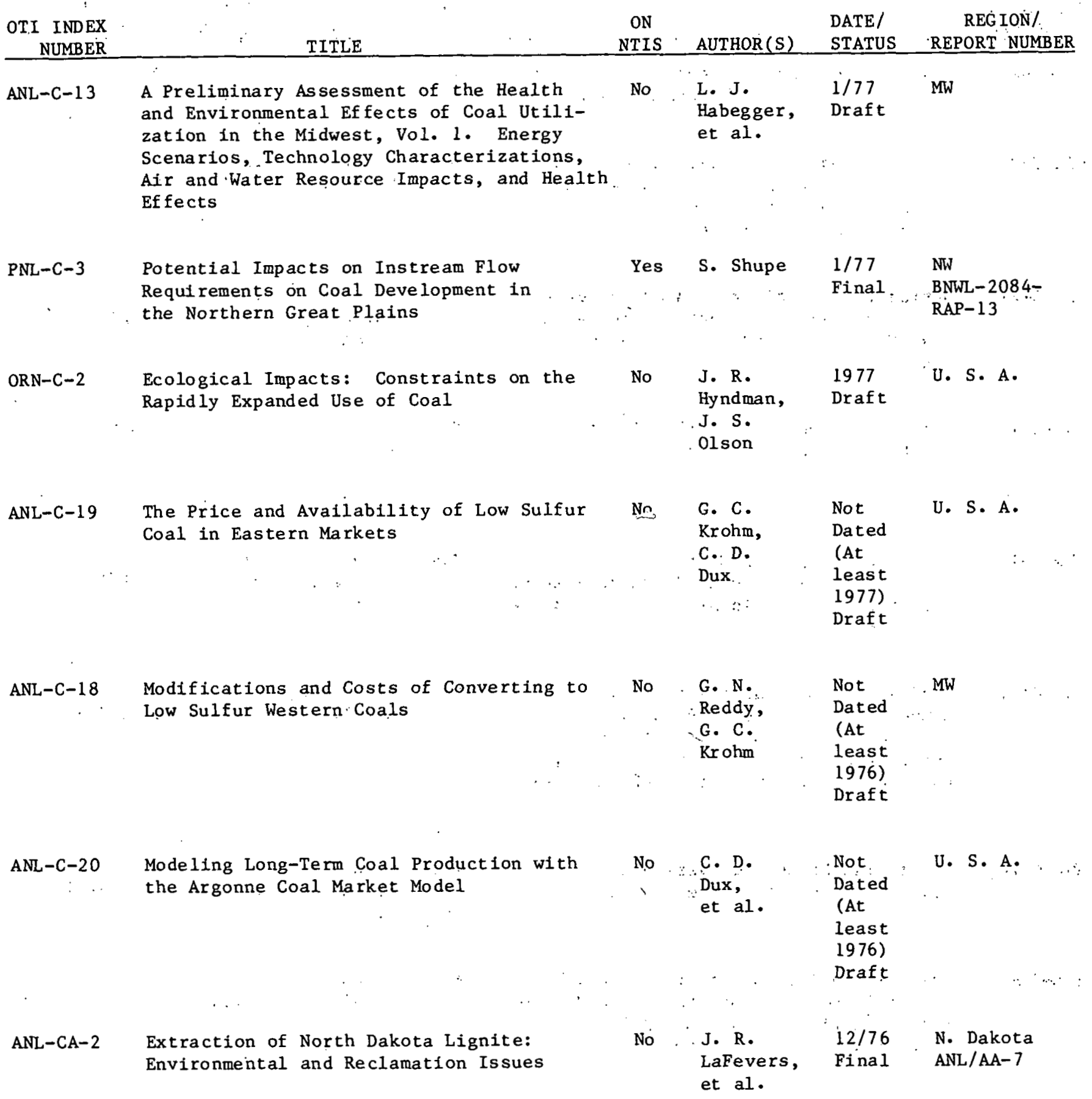


SUBJECT AREA: TECHNOLOGY ASSESSMENT (Coal)

AS OF: APRIL 11, 1978

\begin{tabular}{|c|c|c|c|c|c|}
\hline $\begin{array}{l}\text { OTI INDEX } \\
\therefore \text { NUMBER } \\
\end{array}$ & TITLE & $\begin{array}{c}\text { ON } \\
\text { NTIS }\end{array}$ & AUTHOR (S) & $\begin{array}{l}\text { DATE/ } \\
\text { STATUS } \\
\end{array}$ & $\begin{array}{c}\text { REGION/ } \\
\text { REPORT NUMBER }\end{array}$ \\
\hline PNL-CA- 1 . & $\begin{array}{l}\text { Regional Air Quality Assessment for } \\
\text { Probable Near-Term Coal-Related } \\
\text { Energy Development in the Northwest }\end{array}$ & Yes & $\begin{array}{l}\text { D. Renne, } \\
\text { D. Elliot }\end{array}$ & $\begin{array}{l}10 / 76 \\
\text { Final }\end{array}$ & $\begin{array}{l}\text { NW } \\
\text { BNWL-2084- } \\
\text { RAP-9 }\end{array}$ \\
\hline $\mathrm{PNL}-\mathrm{CA}-2$ & $\begin{array}{l}\text { The Potential for Producing and } \\
\text { Marketing Gasoline Substitutes } \\
\text { from Western Coal }\end{array}$ & Yes & $\begin{array}{l}\text { J. W. } \\
\text { Currie, } \\
\text { D. J. } \\
\text { Braun }\end{array}$ & $\begin{array}{l}9 / 76 \\
\text { Final }\end{array}$ & $\begin{array}{l}\text { NW, RM } \\
\text { BNWL-2084- } \\
\text { RAP-4 }\end{array}$ \\
\hline$\therefore$ & $\cdot$ & & . & & $\cdot \quad:$ \\
\hline $\mathrm{ANL}-\mathrm{CA}-3$ & $\begin{array}{l}\text { A Socioeconomic Assessment of Energy } \\
\text { Developinent in a Small Rural County: } \\
\text { Coal Gasification in Mercer, County, } \\
\text { North Dakota. Vol. 2, }\end{array}$ & No & $\begin{array}{l}\text { T. E. } \\
\text { Baldwin, } \\
\text { et al. }\end{array}$ & $\begin{array}{l}8 / 76 \\
\text { Final }\end{array}$ & $\begin{array}{l}\text { N. Dakota } \\
\text { ANL/AA- } 5\end{array}$ \\
\hline PNL-C-4 & $\begin{array}{l}\text { Dynamic Analysis of the Environmental } \\
\text { and Social Impacts of Coal Development } \\
\text { in the Eastern Powder River Basin of } \\
\text { Wyoming, } 1960-2010\end{array}$ & Yes & $\begin{array}{l}\mathrm{J} \cdot \mathrm{J} \\
\mathrm{Jacobsen}\end{array}$ & $\begin{array}{l}7 / 76 \\
\text { Final }\end{array}$ & $\begin{array}{l}\text { RM } \\
\text { BNWL-2084- } \\
\text { RAP-3 }\end{array}$ \\
\hline ANL-C-17 & $\begin{array}{l}\text { Assessment of Trace Element Body Burdens } \\
\text { Due to Projected Coal Utilization in the } \\
\text { Illinois River Basin }\end{array}$ & No & $\begin{array}{l}\text { J. R. } \\
\text { Gasper, } \\
\text { P. A. } \\
\text { Dauzvardis }\end{array}$ & $\begin{array}{l}\text { Not } \\
\text { Dated } \\
\text { Draft }\end{array}$ & $\begin{array}{l}\text { Il1: River } \\
\text { Basin }\end{array}$ \\
\hline
\end{tabular}


SUBJECT AREA: TECHNOLOGY ASSESSMENT (Geothermal)

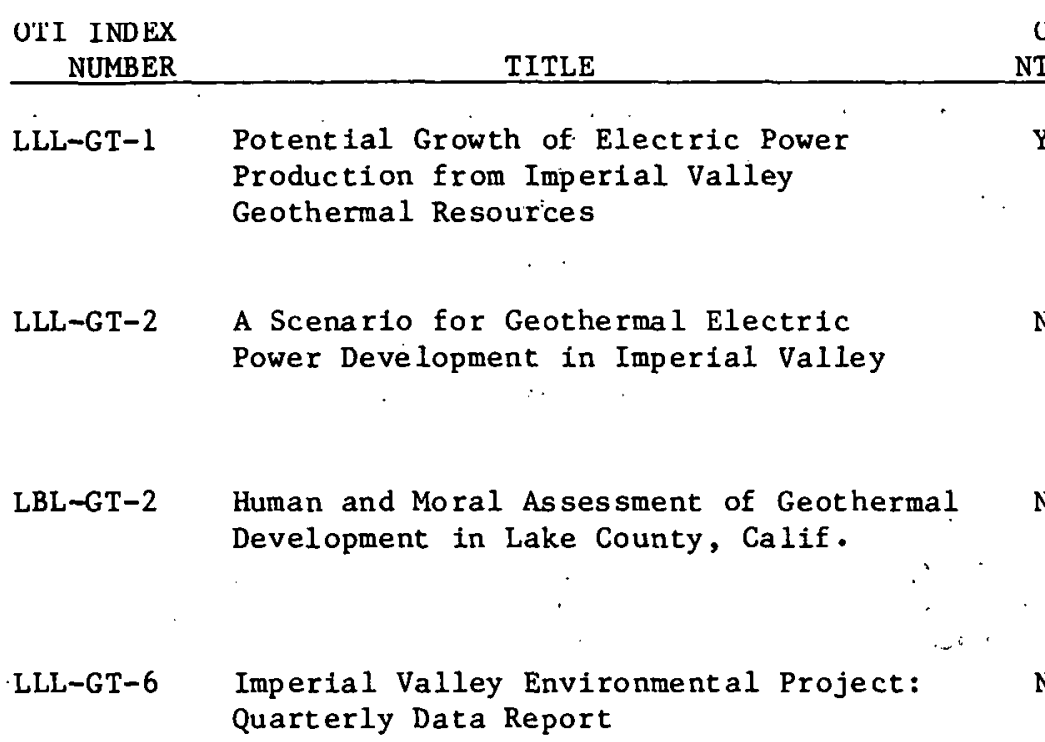

LLL-GT-7 The LLL Geothermal Energy Program Status
Report: January 1976 - January 1977

LLL-GT-10 Geologic.Characteristics of a Portion of the Salton Sea Geothermal Field

LBL-GT-1 76 Lake County Economy: Potential Socioeconomic Impacts of Geothermal Development

LLL-MT-7 On the Geophysical and Geothermal Models of the Earth's Shields and Rift Zones

LLL-GT-6 Potential Effects of Geothermal Energy Conversion on Imperial Valley Ecosystems

LLL-NA-1 A Computational Model of the Stemming Operation for Cased Holes
AS OF: APRIL 11, 1978

DATE/

REGION/

NTIS AUTHOR(S) STATUS REPORT NUMBER

$\begin{array}{llll}\text { Yes } & \begin{array}{l}\text { D. L. } \\ \text { Ermak }\end{array} & \begin{array}{l}9 / 77 \\ \text { Final }\end{array} & \text { SW } \\ \text { UCRL-52252 } \\ \text { No } & \begin{array}{l}\text { D. L. } \\ \text { Ermak }\end{array} & \begin{array}{l}8 / 77 \\ \text { Draft }\end{array} & \begin{array}{l}\text { SW } \\ \text { UCRL-7990-6 } \\ \text { Rev. } 1\end{array}\end{array}$

No

C. W.

$7 / 77$

Churchman, Draft

California

et al.

No

R. Hyholm, 4/77

California

L. R.

Draft

UC ID-17444-1

Anspaugh

Yes

A. L.

11 Austin;

$4 / 77$

Final

California et al.

UCRL-50046-76

. i $\cdot . \cdot$

$\begin{array}{lll}\text { Yes J. D. } & 4 / 77 & \text { California } \\ \text { Tewhey } & \text { Final } & \text { UCRL-52267 }\end{array}$

No L. R. 2/77 California

Vollintine, Final LBL-5944

et al.

$\begin{array}{llll}\text { Yes } & \text { D. H. } & 2 / 77 & \text { U. S. A. } \\ & \text { Chung } & \text { Final } & \text { UCRL-52238 }\end{array}$

$\begin{array}{llll}\text { Yes } & \begin{array}{l}\text { J.H. } \\ \text { Shinn }\end{array} & \begin{array}{l}12 / 76 \\ \text { FInal }\end{array} & \begin{array}{l}\text { California } \\ \text { UCRL-52196 }\end{array} \\ \text { Yes } & \begin{array}{l}\text { S. C. } \\ \text { Cowin }\end{array} & \begin{array}{l}\text { 1/77 } \\ \text { Final }\end{array} & \begin{array}{l}\text { U. S. A. } \\ \text { UCRL-52206 }\end{array}\end{array}$


SUBJECT AREA: TECHNOLOGY ASSESSMENT (Geothermal)

AS OF: APRIL 11, 1978

\begin{tabular}{|c|c|c|c|c|c|}
\hline $\begin{array}{r}\text { OTI INDEXX } \\
\text { NUMBER } \\
\end{array}$ & TITLE & $\begin{array}{c}\text { ON } \\
\text { NTIS }\end{array}$ & $\operatorname{AUTHOR}(\mathrm{S})$ & $\begin{array}{l}\text { DATE/ } \\
\text { STATUS } \\
\end{array}$ & $\begin{array}{l}\text { REGION/ } \\
\text { REPORT NUMBER } \\
\end{array}$ \\
\hline LLL-GT-3 & $\begin{array}{l}\text { The Geysers Geothermal Power Plant: } \\
\text { Environmental Impact of the Release } \\
\text { of } 222 \mathrm{Rn}\end{array}$ & No & $\begin{array}{l}\text { L. R. } \\
\text { Anspaugh, } \\
\text { et al. }\end{array}$ & $\begin{array}{l}1 / 77 \\
\text { Draft }\end{array}$ & $\begin{array}{l}\text { California } \\
\text { UCRL-79038 }\end{array}$ \\
\hline LLL-GT-1 3 & $\begin{array}{l}\text { Potential Ef fects of Geothermal Energy } \\
\text { Conversion on Imperial Valley Ecosystems }\end{array}$ & Yes & $\begin{array}{l}\text { J. H. } \\
\text { Shinn } \\
\text { (ed.) }\end{array}$ & $\begin{array}{l}12 / 76 \\
\text { Final }\end{array}$ & $\begin{array}{l}\text { California } \\
\text { UCRL-52196 }\end{array}$ \\
\hline LLL-GT-4 & $\begin{array}{l}\text { A Description of Imperial Valley, } \\
\text { California for the Assessment of } \\
\text { Impacts of Geothermal Energy Develop- } \\
\text { ment }\end{array}$ & Yes & $\begin{array}{l}\text { D. Layt on, } \\
\text { D. Ermak }\end{array}$ & $\begin{array}{l}8 / 76 \\
\text { Final }\end{array}$ & $\begin{array}{l}\text { California } \\
\text { UCRL-52121 }\end{array}$ \\
\hline LLL-MT-5 & $\begin{array}{l}\text { Imperial Valley Environmental Project: } \\
\text { Progress Report }\end{array}$ & Yes & $\begin{array}{l}\text { P. Phelps, } \\
\text { L. Ans- } \\
\text { paugh } \\
\text { (eds.) }\end{array}$ & $\begin{array}{l}10 / 76 \\
\text { Final }\end{array}$ & $\begin{array}{l}\text { Calif ornia } \\
\text { UCRL-50044- } \\
76-1\end{array}$ \\
\hline LLL-GT-5 & $\begin{array}{c}\text { An Overview of the Imperial Valley } \\
\text { Environmental Project }\end{array}$ & Yes & $\begin{array}{l}\text { L. R. } \\
\text { Anspaugh, } \\
\text { P. L. } \\
\text { Phelps } \\
\text { (eds.) }\end{array}$ & $\begin{array}{l}4 / 76 \\
\text { Draft }\end{array}$ & $\begin{array}{l}\text { California } \\
\text { UCID- } 17067\end{array}$ \\
\hline LLL-MT- 6 & Energy and Technology Review & No & $\begin{array}{l}\text { Not } \\
\text { stated }\end{array}$ & $\begin{array}{l}1976 \\
\text { Final }\end{array}$ & $\begin{array}{l}\text { U. S. A. } \\
\text { UCRL-52000- } \\
76-5\end{array}$ \\
\hline LLL-GT-8 & $\begin{array}{l}\text { Characteristics of Geothermal Wells } \\
\text { Located in the Salton Sea Geothermal } \\
\text { Field, Imperial County, California }\end{array}$ & Yes & $\begin{array}{l}\text { T. D. } \\
\text { Palmer }\end{array}$ & $\begin{array}{l}12 / 75 \\
\text { Final }\end{array}$ & $\begin{array}{l}\text { Calif ornia } \\
\text { UCRL-51976 }\end{array}$ \\
\hline LLL-GT -9 & $\begin{array}{l}\text { Present Status and Future Prospects } \\
\text { for Nonelectrical Uses of Geothermal } \\
\text { Resources }\end{array}$ & Yes & $\begin{array}{l}\text { J. H. } \\
\text { Howard, } \\
\text { ed. }\end{array}$ & $\begin{array}{l}10 / 75 \\
\text { Fina } 1\end{array}$ & $\begin{array}{l}\text { Global } \\
\text { UCRL-51926 }\end{array}$ \\
\hline LLL-GT-1 2 & $\begin{array}{l}\text { Potential Power Generation \& Gas } \\
\text { Production from Gulf Coast Geo- } \\
\text { pressure Reservoirs }\end{array}$ & Yes & $\begin{array}{l}\text { P. A. } \\
\text { House, } \\
\text { et al. }\end{array}$ & $\begin{array}{l}5 / 75 \\
\text { Final. }\end{array}$ & $\begin{array}{l}\text { Texas } \& \\
\text { Louisiana } \\
\text { Gulf Coast }\end{array}$ \\
\hline
\end{tabular}


SUBJECT AREA: TECHNOLOGY ASSESSMENT (EDP's)

OTI INDEX NUMBER

DOE-N-4 Mining and Milling EDP

DOE-NA-2 Uranium Enrichment Program EDP

DOE-MT-3 Energy Technology Characteristics

for Section 13(A) Water Assessments

DOE-NG-1 Enhanced Gas Recovery EDP

DOE-OL-1 Oil Supply EDP

DOE-CA-5 Magnetohydrodynamics Program EDP

DOE-N-1 Decontamination and Decommissioning EDP

DOE-NA-3 Space Applications EDP

DOE-NA-4 Magnetic Fusion Program EDP
AS OF: APRIL 11,1978

\begin{tabular}{|c|c|c|c|}
\hline $\begin{array}{c}\text { ON } \\
\text { NTIS } \\
\end{array}$ & AUTHOR(S) & $\begin{array}{l}\text { DATE/ } \\
\text { STATUS }\end{array}$ & $\begin{array}{c}\text { REGION/ } \\
\text { REPORT NUMBER }\end{array}$ \\
\hline No & Not stated & $\begin{array}{l}2 / 78 \\
\text { Draft }\end{array}$ & U. S. A. \\
\hline No & Not stated & $\begin{array}{l}1 / 78 \\
\text { Draft }\end{array}$ & U. S. A. : \\
\hline No & Not stated & $\begin{array}{l}1 / 78 \\
\text { Fina } 1\end{array}$ & $\begin{array}{l}\text { Missouri, } \\
\text { Colorado, } \\
\text { and Ohio } \\
\text { River Basins }\end{array}$ \\
\hline No & $\begin{array}{l}\text { C. B. } \\
\text { Wilson, } \\
\text { R. Franklin }\end{array}$ & $\begin{array}{l}12 / 77 \\
\text { Draft }\end{array}$ & U. S. A. \\
\hline No & $\begin{array}{l}\text { C. B. } \\
\text { Wilson, } \\
\text { R. Franklin }\end{array}$ & $\begin{array}{l}11 / 77 \\
\text { Draft }\end{array}$ & U. S. A. \\
\hline
\end{tabular}

No G. Rotariu, $10 / 77$ U. S. A. J. Siegel Draft

No

A. F.

$9 / 77$

U. S. A.

Kluk, Draft

C. M.

Campbel1

No

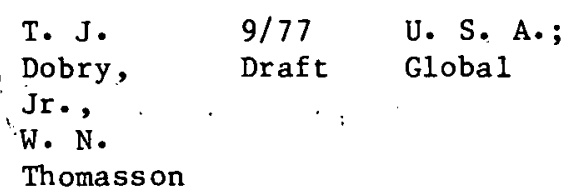

No

$\begin{array}{ll}\text { J. E. } & 9 / 77 \\ \text { Baublitz, } & \text { Draft } \\ \begin{array}{l}\text { R. P. } \\ \text { Blaunstein }\end{array} & \end{array}$


SUBJECT AREA: TECHNOLOGY ASSESSMENT (EDP's)

AS OF: APRIL 11, 1978

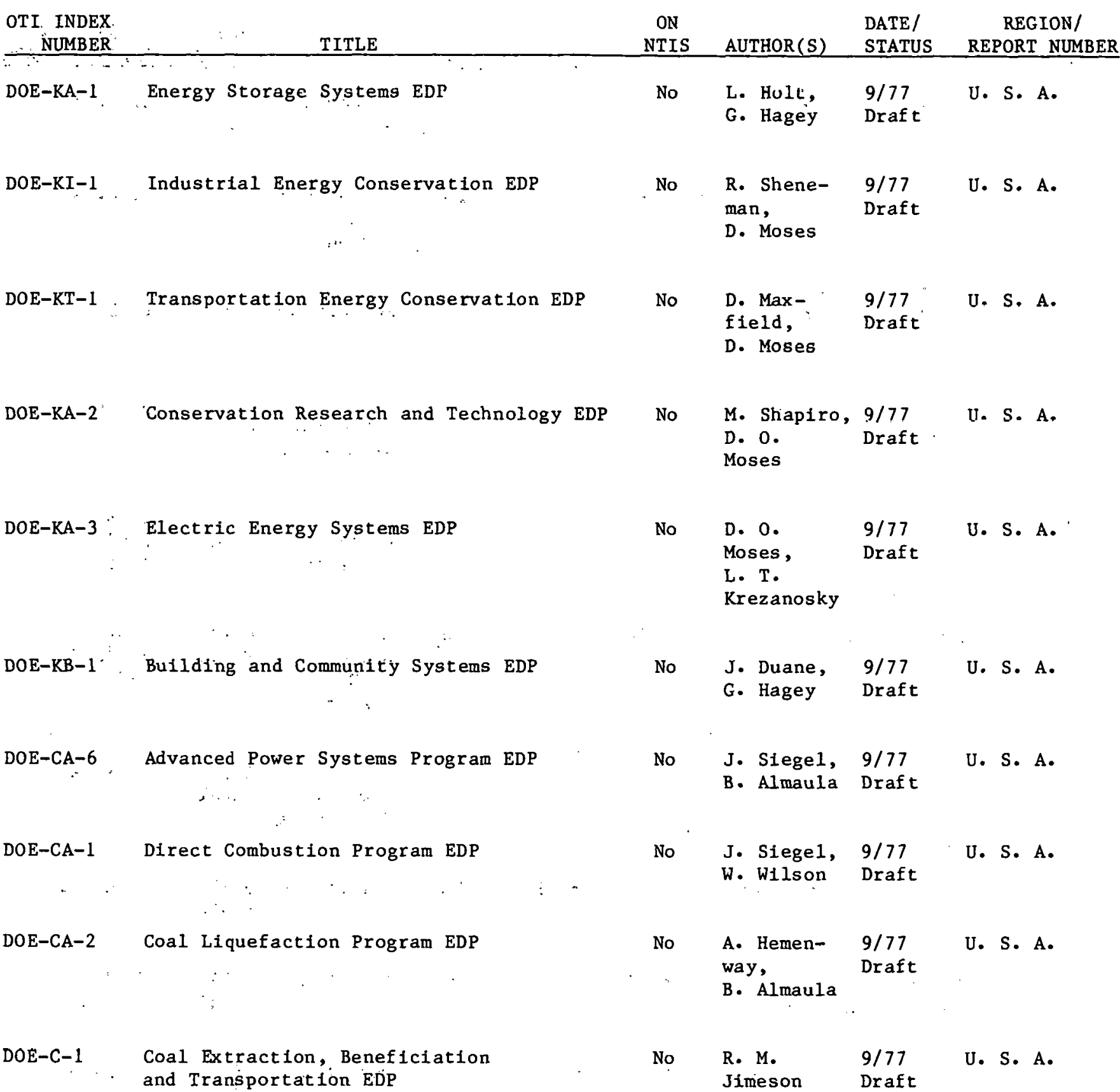


SUBJECT AREA: 'TECHNOLOGY ASSESSMENT (EDP's)

AS OF: APRIL $11,1978^{\circ}$

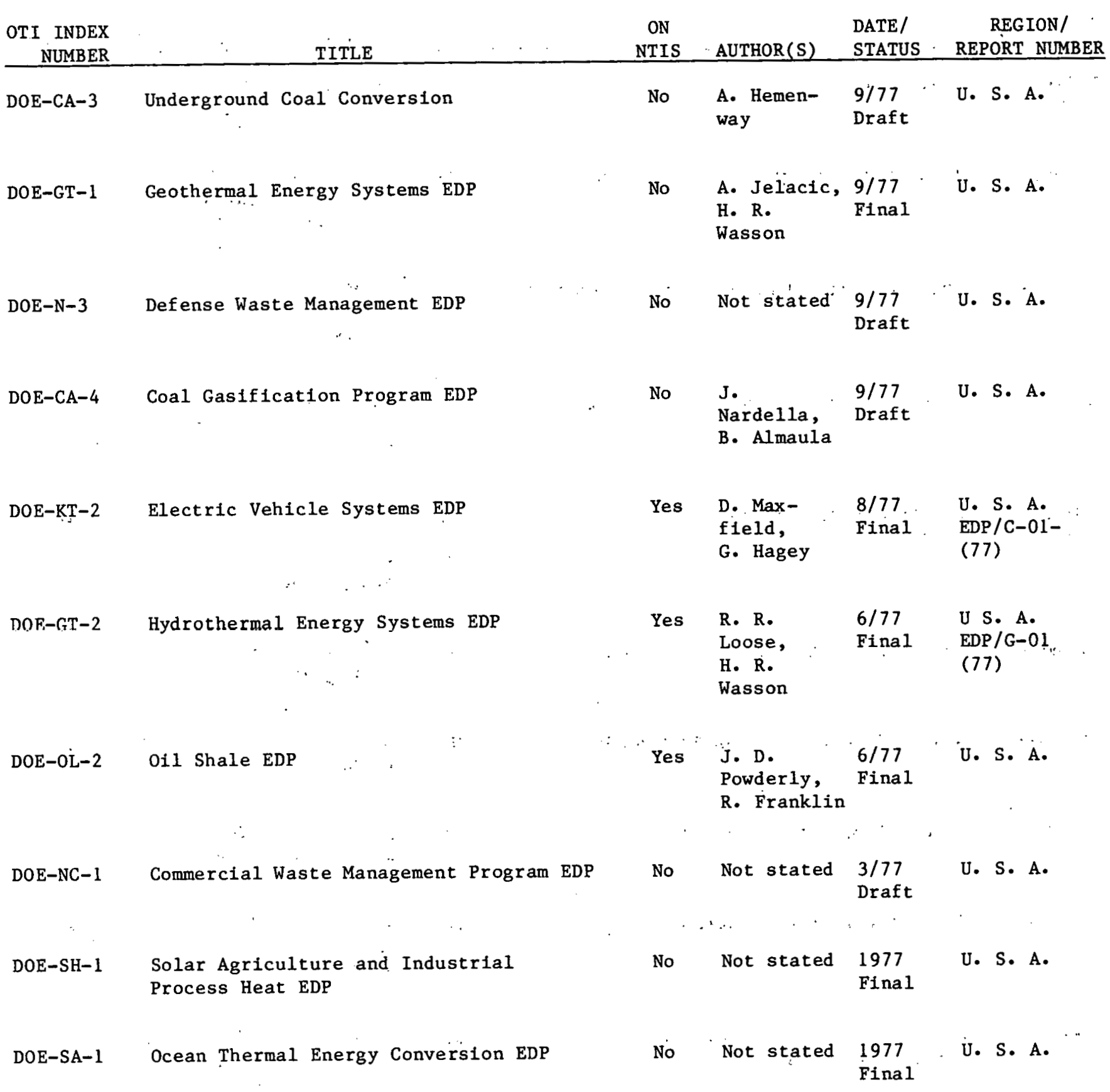


SUBJECT AREA: TECHNOLOGY ASSESSMENT (EDP's)

OTI INDEX NUMBER TITLE

DOE-SA-2 Fuels from Biomass EDP

DOE-SA-3 Wind Energy Conversion EDP

DOE-SA-4

Solar Thermal Power Systems EDP

DOE-SA-5 Photovoltaics EDP DOE-SH-2
EDP

DOE-N-2 Special Nuclear Materials Production

DOE-NA-1 Advanced Isotope Separation EDP
AS OF: APRIL 11,1978

DATE/ REGION/

NTIS AUTHOR(S) STATUS REPORT NUMBER

No Not stated 1977 U. S. A.

No $\underset{\text { EEAI }}{\text { Not stated }} \underset{\text { Draft }}{1977}$ U. S. A.

No Not stated $\begin{array}{ll}.1977 \\ \text { EEAI U. S. A. }\end{array}$

No Not stated $1977 \quad$ U. S. A.

EEAI Final

No Not stated $1977 \quad$ U. S. A. EEAI Final

No Not stated No date U. S. A. Draft

No Not, No date U. S. A. stated. Draft. 
OFFICE OF TECHNOLOGY IMPACTS INVENTORY OF REPORTS

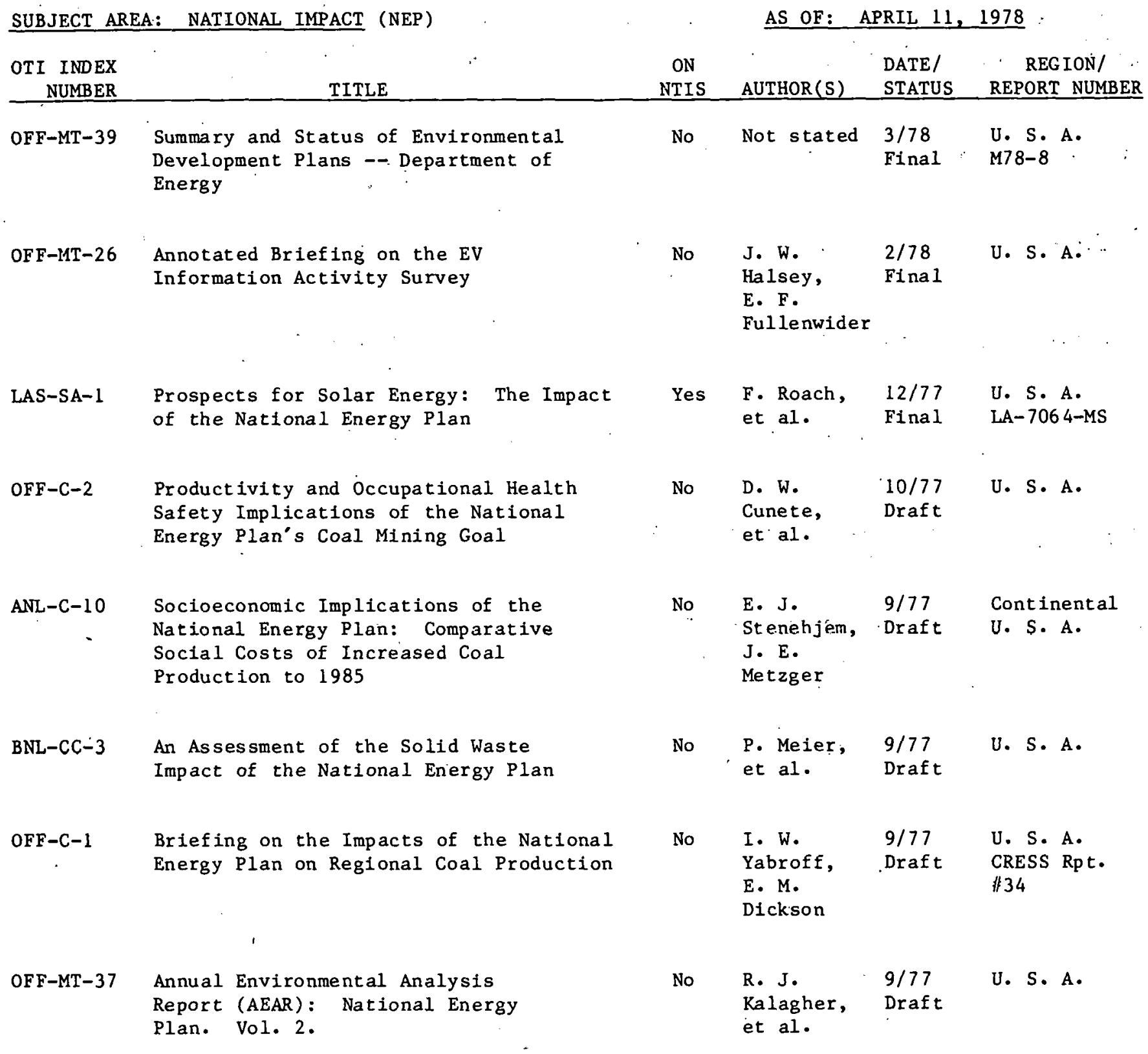




\begin{tabular}{|c|c|c|c|c|c|}
\hline $\begin{array}{r}\text { OTI INDEX } \\
\text { NUMBER } \\
\end{array}$ & TITLE & $\begin{array}{c}\text { ON } \\
\text { NTIS }\end{array}$ & AUTHOR(S) & $\begin{array}{l}\text { DATE/ } \\
\text { STATUS }\end{array}$ & $\begin{array}{l}\text { REG ION/ } \\
\text { REPORT NUMBER } \\
\end{array}$ \\
\hline OFF-MT-38 & $\begin{array}{l}\text { Annual Environmental Analysis } \\
\text { Report (AEAR): National Energy } \\
\text { Plan. Vol. } 4 .\end{array}$ & No & $\begin{array}{l}\text { R. J. } \\
\text { Kalagher, } \\
\text { et al. }\end{array}$ & $\begin{array}{l}9 / 77 \\
\text { Draft }\end{array}$ & U. S. A. \\
\hline OFF-MT-1 & $\begin{array}{l}\text { Annual Environmental Analysis } \\
\text { Report (AEAR): National Energy } \\
\text { Plan. Vol. 1. }\end{array}$ & No & $\begin{array}{l}\text { R. J. } \\
\text { Kalagher, } \\
\text { et al. }\end{array}$ & $\begin{array}{l}9 / 77 \\
\text { Draft }\end{array}$ & $\begin{array}{l}\text { U. S. A. } \\
\text { MTR- } 7626\end{array}$ \\
\hline ANL-MT-6 & $\begin{array}{l}\text { Water Pollution Impacts of the National } \\
\text { Energy Plan }\end{array}$ & No & $\begin{array}{l}\text { L. Habeg- } \\
\text { ger, } \\
\text { et al. }\end{array}$ & $\begin{array}{l}8 / 77 \\
\text { Draft }\end{array}$ & $\begin{array}{l}\text { Continental } \\
\text { U. S. A. }\end{array}$ \\
\hline$B N L-M T-3$ & $\begin{array}{l}\text { Preliminary Assessment of the Impacts } \\
\text { of the National Energy Plan on the } \\
\text { Northeast }\end{array}$ & No & $\begin{array}{l}\text { P. Meier, } \\
\text { et al. }\end{array}$ & $\begin{array}{l}8 / 77 \\
\text { Draft }\end{array}$ & NE \\
\hline BNL-CC-4 & $\begin{array}{l}\text { Preliminary Assessment of the Solid } \\
\text { Waste Management Impact of the National } \\
\text { Energy Plan }\end{array}$ & No & $\begin{array}{l}\text { P. Meier, } \\
\text { et al. }\end{array}$ & $\begin{array}{l}8 / 77 \\
\text { Draft }\end{array}$ & U. S. A. \\
\hline ORN-MT- 17 & $\begin{array}{l}\text { Nationwide Assessment of Water Quantity } \\
\text { Impacts of the National Energy Plan. } \\
\text { Vol. 1. Summary and Conclusions }\end{array}$ & Yes & $\begin{array}{l}\text { J. E. } \\
\text { Dobson, } \\
\text { et al. }\end{array}$ & $\begin{array}{l}8 / 77 \\
\text { Final }\end{array}$ & $\begin{array}{l}\text { U. S. A. } \\
\text { ORNL-TM-6098 }\end{array}$ \\
\hline OF F-MT-25 & $\begin{array}{l}\text { Annotated Briefing on the AES } \\
\text { Information Activity Survey }\end{array}$ & No & $\begin{array}{l}\text { E. D. } \\
\text { Fullenwider }\end{array}$ & $\begin{array}{l}8 / 77 \\
\text { Final }\end{array}$ & U. S. A. \\
\hline OFF-MT -5 & $\begin{array}{l}\text { Inventory of Federal Energy-Related } \\
\text { Environment and Safety Research for } \\
\text { FY } 1976 . \text { Vol. } 4 .\end{array}$ & Yes & $\begin{array}{l}\text { Aerospace } \\
\text { Corp. }\end{array}$ & $\begin{array}{l}4 / 77 \\
\text { Final }\end{array}$ & $\begin{array}{l}\text { U. S. A. } \\
\text { ERDA } 77-50 / 4\end{array}$ \\
\hline BNL-MT-19 & $\begin{array}{l}\text { Regional Disaggregation of ERDA's } \\
\text { Forecast } 2 \text { Energy Scenario for the } \\
\text { Year } 2000\end{array}$ & No & $\begin{array}{l}\text { R. J. } \\
\text { Goettle, } \\
\text { J. } \\
\text { Brainard, } \\
\text { I. Bhas in }\end{array}$ & $\begin{array}{l}3 / 77 \\
\text { Draft }\end{array}$ & $\begin{array}{l}\text { U. S. A. } \\
\text {. }\end{array}$ \\
\hline
\end{tabular}




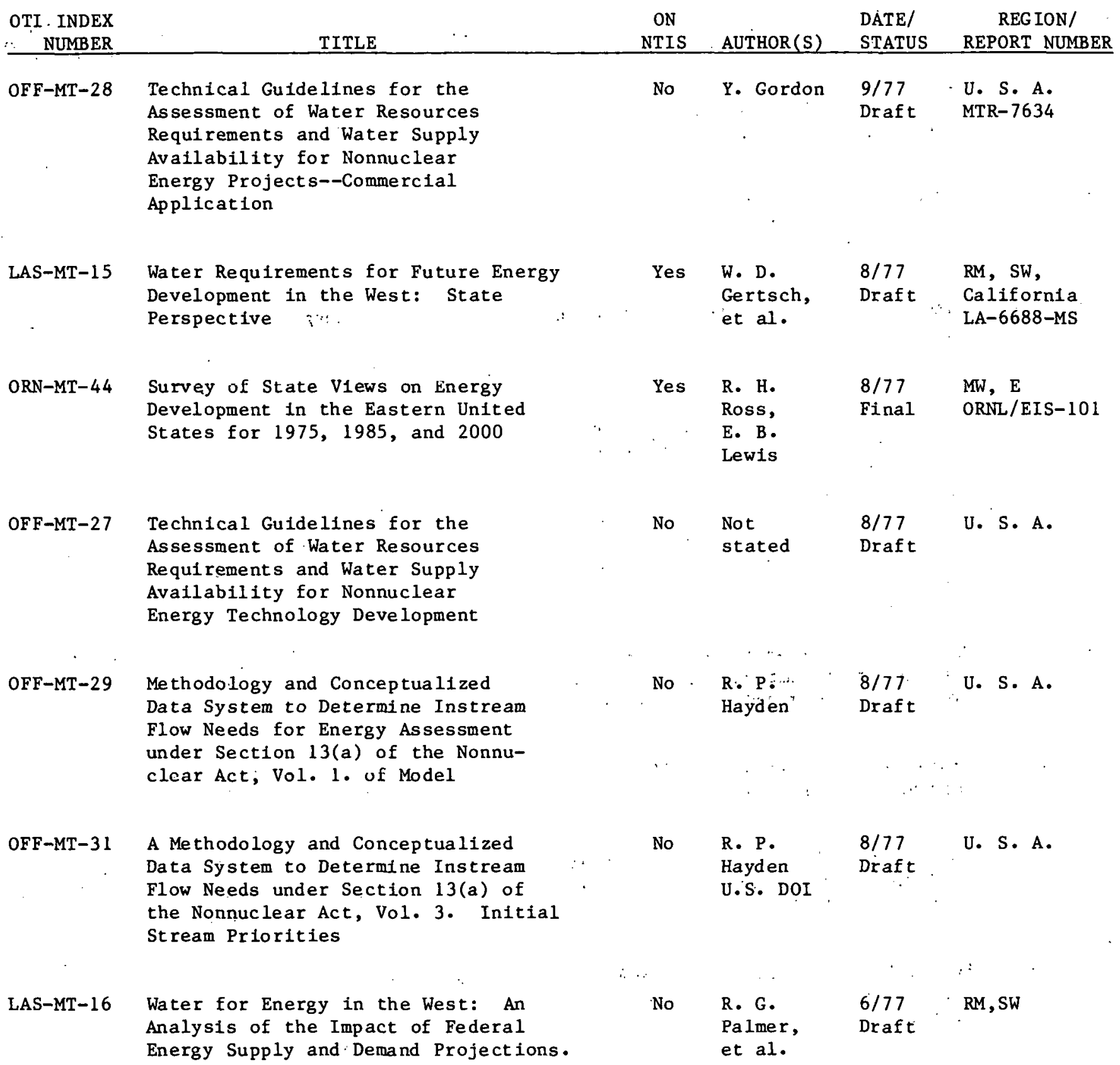




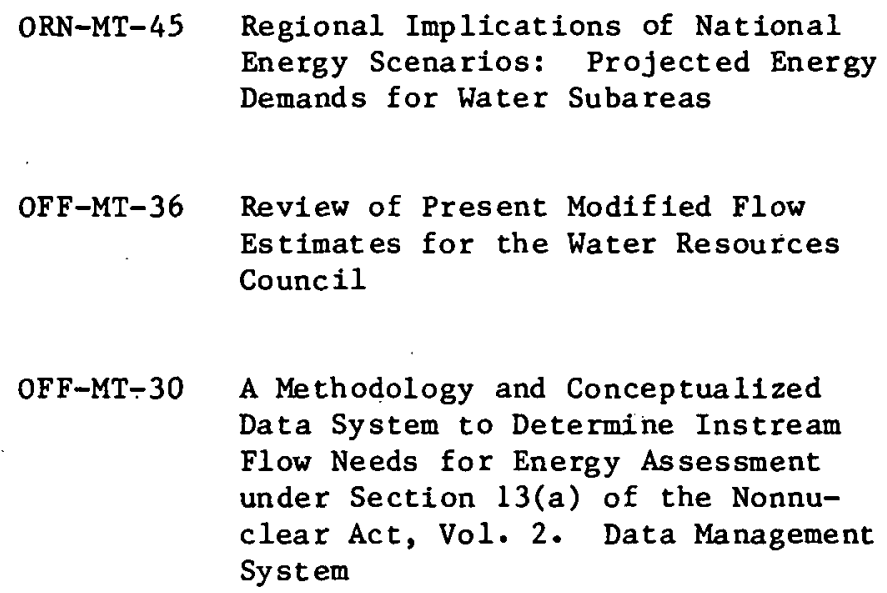

Demands for Water Subareas

OF F-MT-36

Review of Present Modified Flow Estimates for the Water Resources Council

$5 / 77$

U. S. A.

Draft

$\begin{array}{llll}\text { No } & \text { U. of } & 5 / 77 & \text { Selected } \\ \text { Texas } & \text { Final } & \text { RIver Basins }\end{array}$

OFF-MT-35 Water for Energy: Case Study Assessments in Appalachia

OFF-MT-34

Prelfminary Survey of Federal Water Quallty Data Bases for Use in Assessing Impacts of Energy Development

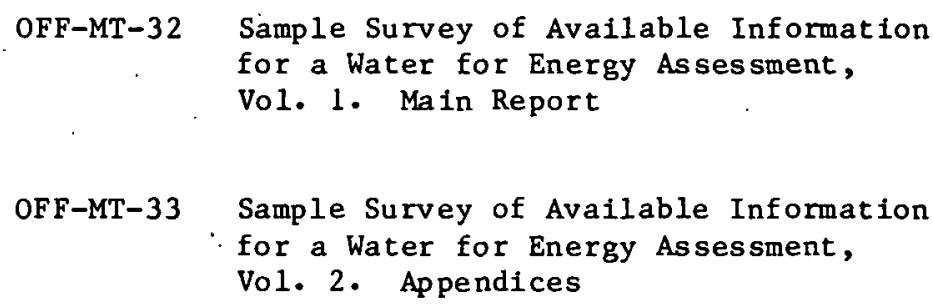

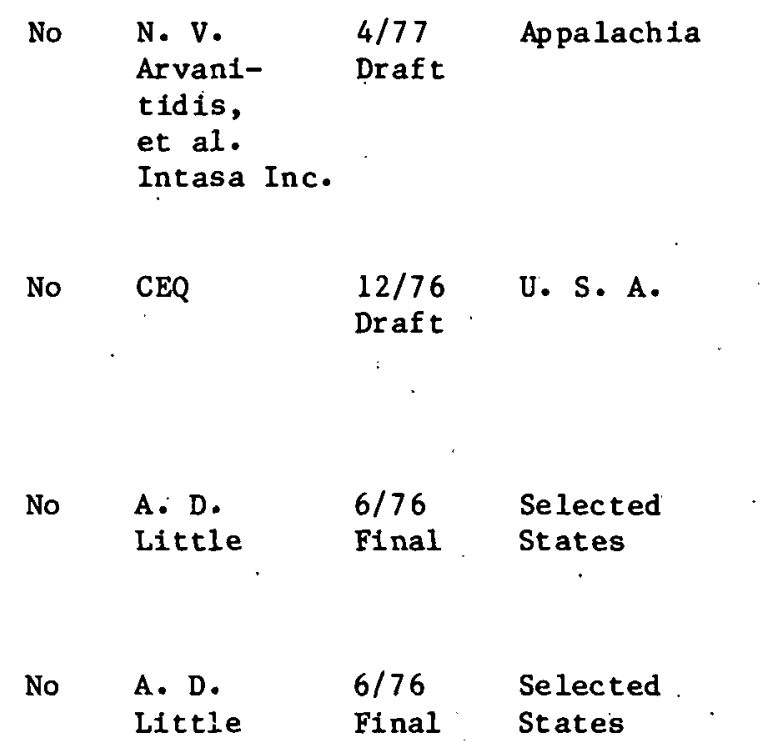




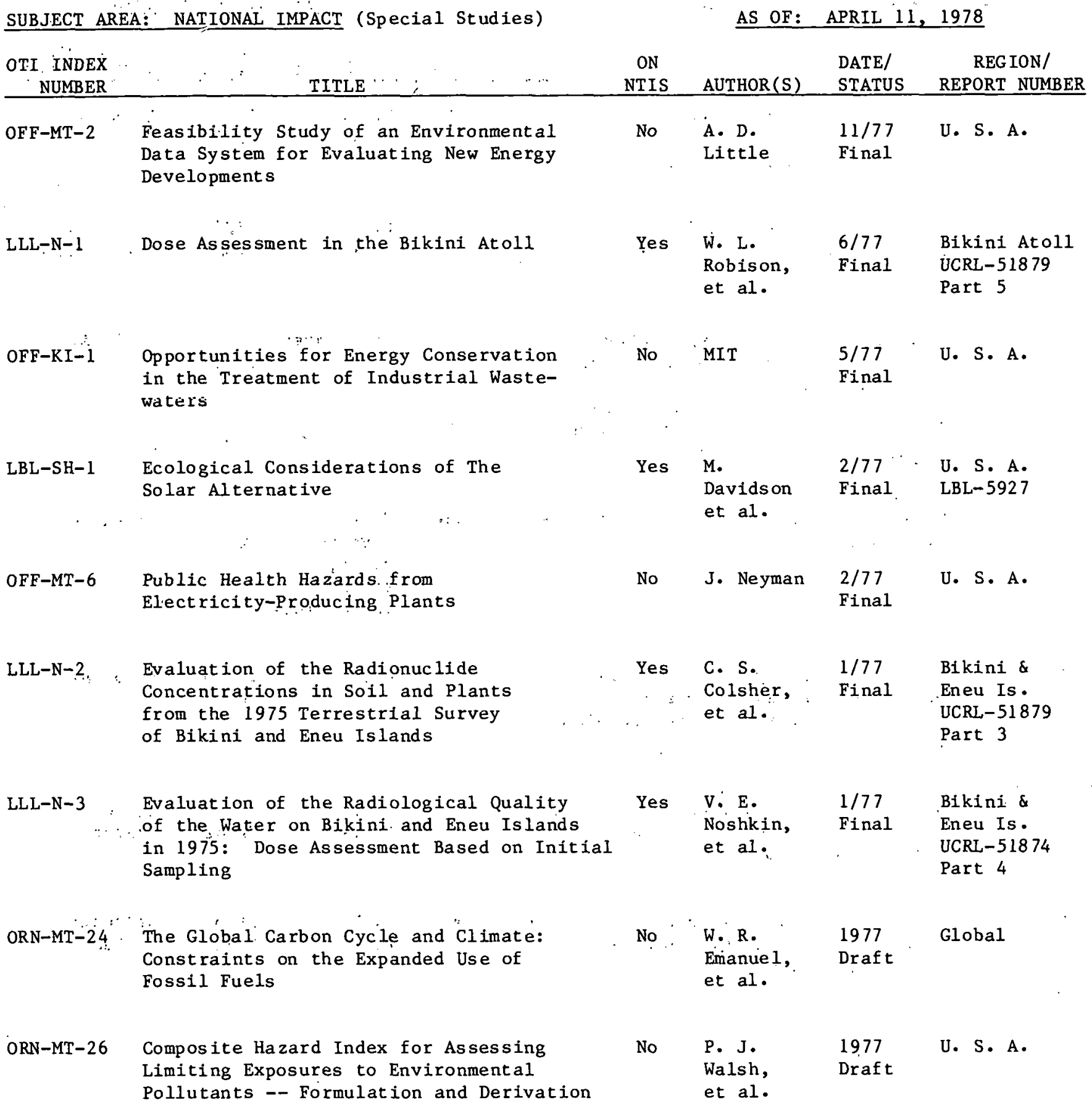


SUBJECT AREA: NATIONAL IMPACT (Special Sțudies)

AS OF: APRIL 11, 1978

OTI INDEX NTIS

National Plan for Energy Research, Development and Demonstration. Sixth Public Meeting

LLL-N-4 Derivation of Plant-Soil Relationships for Dose Assessment on Bikini Atoll

OFF-MT -8

A National Plan for Energy Research, Development and Demonstration. Third Public Meeting

ORN-MT-25

Optimal Siting of Energy Facilities for Minimum Air Pollutant Exposure on a Regional Scale
ON

No

\begin{tabular}{|c|c|c|}
\hline No & Not stated & $\begin{array}{l}12 / 76 \\
\text { Final }\end{array}$ \\
\hline Yes & $\begin{array}{l}\text { C. S. } \\
\text { Colsher }\end{array}$ & $\begin{array}{l}11 / 76 \\
\text { Draft }\end{array}$ \\
\hline No & Not stated & $\begin{array}{l}5 / 76 \\
\text { Final }\end{array}$ \\
\hline No & $\begin{array}{l}\text { W. R. } \\
\text { Emanuel, } \\
\text { et al. }\end{array}$ & Draft \\
\hline
\end{tabular}

REGION/ REPORT NUMBER

U. S. A. ERDA $-71 / 1$ (PM-6)

ikini Atoll UCID-17313

. S. A. ERDA-76/1 (PM-3) et al. 
OFFICE OF TECHNOLOGY IMPACTS INVENTORY OF REPORTS

SUBJECT AREA: REGIONAL IMPACTS (Northeast)
BNL-MT-4 A Perspective on the Energy Future of the Northeast United States

BNL-MT-18 Mid-Atlantic Briefing Document

BNL-MT-6 Annual Report for, Fiscal Year 1976

BNL-MT-7 The Energy Situation in New England

BNL-MT-12 A Perspective on the Energy Future of the Northeast: Health and Environmental Impacts of Alternative Energy Futures for the Northeast.
AS OF: APRIL 11,1978

No

D. Dixon-

$7 / 77$

Draft Davis, et al.

Yes

J. Brain-

ard,

$6 / 77$

NE et al.

Final BNL-50550

et alo

No

\section{Manson}

1977

Brainard

Draft

NE

BNL-50703

Yes

P. M;

Meier

$12 / 76$

Final.

NE

BNL-50668

Yes

J. P. Brainard,

$11 / 76$

Final

NE et al.

L. D. $3 / 76$ Hamilton, Draft NE et al.

W. Virginia $-50703$

BNL -50580

No
et al. 
SUBJECT AREA: REGIONAL IMPACTS (Midwest)

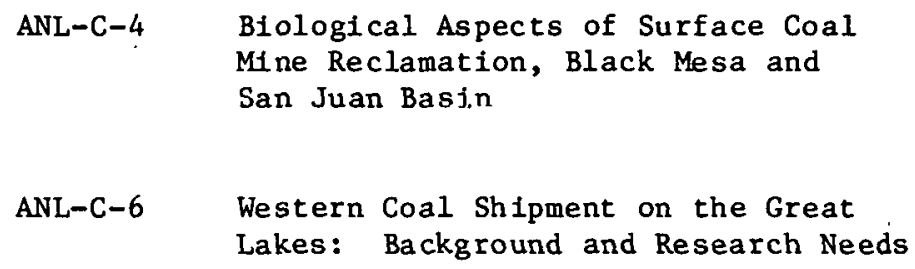

ANL-MT-4 Regional Comparative Assessment - Work . St atement

ANL-CA-7 Survey of Federal and Selected State Laws and Regulations Relevant to Coal Fired MHD Power Plants: A Support Document to the EDP of the Magnetohydrodynamics Program of the Department of Energy

ANL-MT-29

Environmental Status of the Lake Michigan Region, Vo1. 14. Birds of the Lake Michigan Drainage Basin

$\begin{array}{ll}\text { ANL-CA-5 Social Cost's for Alternative High-BTU } & \\ & \text { Gas Supply Systems Incomplete Copy }\end{array}$

ANL-C-11 Assessment of the Health and Environmental Effects of Power Generation in the Midwest, Vo1. 2. Ecological Effects

ANL-MT-5 A Model for Predicting Fish Impingement at Cooling Water Intakes

ANL-MT-7 A Framework for Detailed Site-Specific Studies of Local Socio-economic Impacts from Energy Development
AS OF: APRIL 11, 1978

$\begin{array}{lll}\text { No B. Green } & 12 / 77 & \text { Arizona \& } \\ \text { Final } & \text { New Mexico }\end{array}$

No

L. R. Lewis,

$10 / 77$

Final

MW \& Western

R. C.

Stupka

No

None

Given

$10 / 77$

Final

No

M. Sauer

10/77

Final

Selected

States and

USA in

Genera 1

Yes

G. J.

$7 / 77$

MW

Wallace

Final

ANL/ES-40

No

None

$6 / 77$

Draft

NW \& Gulf

stated

No

A. J.

4/77

Draft

MW et al.

Yes

I. P.

Murarka

$1 / 77$

Final

Generic

ANL/AA-8

No

T. E.

$12 / 76$

N. Dakota

Baldwin, Draft

\& USA 
SUBJECT AREA: REGIONAL IMPACTS (Midwest)

ANL-MT-8 A Framework for Comparative Analysis of Sociloeconomic Impact: CASE-I

ANL-MT-9' Present Energy Problems 'and Future Trends in the Midwest

ANL-MT-10

Environmental Status of the Lake Michigan Region; Vol. 4. Phytoplankton of Lake Michigan

ANL-MT-1 1

An Evaluation of Regional Trends in Power Plant Siting and Energy Transport

ANL-C-3 Modeling Long-Term Regional Coal Production

ANL-MT-12 Environmental Status of the Lake Michigan Region, Vol. 8. Atmospheric Environment of the Lake Michigan Drainage Basin

ANL-MT-27. Bibliography of Energy-Related Integrated Assessments

ANL-MT-13 Environmental Status of the Lake Michigan Region. Vol. 17. Inland
AS OF: APRIL 11,1978

No

E. J.

$12 / 76$ Stenehjem, Draft et al.

No

G. C.

$12 / 76$

Krohm,

Draft

et al.

Yes

No

No

Yes

No

M. Hilton

No
S. J.

$12 / 76$

Tarapchak, Final

E. F.

Stoermer

R. R:

$12 / 76$

Cirillo,

Draft

et al.

C. D. Dux,

et al.

$11 / 76$

Draft

D. F. Gatz, 11/76

S. A.

Final

Changnon,

Jr.

$11 / 76$

Draft

Generic

G. C.

Becker

$9 / 76$

Draft

MW: Lake

Michigan

ANL/ES-40

T. E

Baldwin,

$8 / 76$

Draft

N. Dakota

MW: Lake ANL/ES-40

MW: Lake Michigan ANL/ES-40 et al. Coal Gastfication in Mercer County, North Dakota, Volume 2 
Socioecononic Assessment of Energy Development in a Small Rural County: Coal Gasification in Mercer County, N. Dakota, Vol. 1

ANL-MT-14

Environmental Status of the Lake Michigan Region, Vol. '16. Amphibians and Reptiles of the Lake Michigan Drainage Basin

ANL-MT-15 A Long-Term Econometric Analysis of Electricity Demand in the Ohio River Basin

ANL-MT-16

Environmental status of the Lake Michigan Region, Vol. 3. Chemistry of Lake MichIgan

ANL-MT-17 Forecasting the Local Economic Impacts of Energy Resource Development: A Methodological Approach

ANL-MT-19

Social Cost Studies Program. Electrical Utillty Generating Sysțem Reliability Analysis Code, SYSREL

ANL-MT- 1

SITE: A Methodology for Assessment of Energy Facility Siting Patterns

ANL-MT-20 Environmental Status of the Lake Michigan Region, Vol. 2. Physical Limnology of Lake Michigan. Part 1. Physical Characteristics. Part 2. D.ffusion and Dispersion
No

Yes

$\begin{array}{lll}\text { K. W. } & 8 / 76 & \text { Generic } \\ \text { Costello } & \text { Draft } & \end{array}$

T. E.

$8 / 76$

N. Dakota Baldwin,

Final ANL/AA-5 et al.

\begin{tabular}{|c|c|c|c|}
\hline Yes & $\begin{array}{l}\text { E. D. } \\
\text { Pentecost, } \\
\text { R. C. }\end{array}$ & $\begin{array}{l}7 / 76 \\
\text { Final }\end{array}$ & $\begin{array}{l}\text { NW: Lake } \\
\text { MIchigan } \\
\text { ANL/ES-40 }\end{array}$ \\
\hline
\end{tabular}

$\begin{array}{lll}\text { No G. C. } & 5 / 76 & \text { Ill., Ind., } \\ \text { Krohm, } & \text { Draft } & \text { Ohio, Penn., } \\ \text { et al. } & & \text { Ky., Tenn., } \\ & \text { W. Va. }\end{array}$

$\begin{array}{llll}\text { Yes } & \text { M. S. } & 5 / 76 & \text { MW: Lake } \\ \text { Torrey } & \text { Final } & \text { Michigan } \\ & & & \text { ANL/ES-40 }\end{array}$

\begin{tabular}{|c|c|c|c|}
\hline Yes & $\begin{array}{c}\text { E. J. } \\
\text { Stenehjem } \\
.\end{array}$ & $\begin{array}{l}12 / 75 \\
\text { Final }\end{array}$ & $\begin{array}{l}\text { Generic } \\
\text { ANL/AA-3 }\end{array}$ \\
\hline Yes & $\begin{array}{l}\text { K. Hub, } \\
\text { et al. }\end{array}$ & $\begin{array}{l}9 / 75 \\
\text { Final }\end{array}$ & $\begin{array}{l}\text { Generic } \\
\text { ANL/AA-4 }\end{array}$ \\
\hline
\end{tabular}
No N. A. 8/75 Generic Frigerio, Final ANL/AA-2 et al.

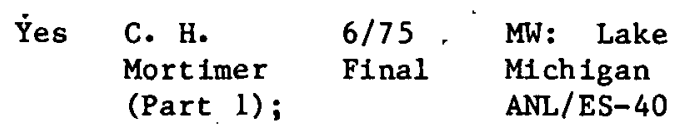

G. T. Cs anady (Part 2) 
SUBJECT AREA: REGIONAL IMPACTS (Midwest)

ANL-MT-25 Regional Energy Mndeling: All Evaluation of Alternative Approaches

ANL-MT-26 Regional Studies Advisory Board Research Recommendations

ANL-MT-21 Environmental Status of the Lake Michigan Region, Vol. 10. Vegetation of the Lake Michigan Drainage Basin

ANL-MT-22 Environmental Status of the Lake Michigan Region, Vol.. 9. Solls of the Lake Michigan Drainage Basin an Overview

ANL-MT-23 Environmental Status of the Lake Michigan Region, Vol.. 15. Mammals of the Lake Michigan Drainage Basin

ANL-MT-24. Environmental Status of the Lake Mich Igan Region, Vo1. 7. Earthquake History and Measurement with Application to the Lake Michigan Drainage Basin

\section{AS OF: APRIL 11, 1978}

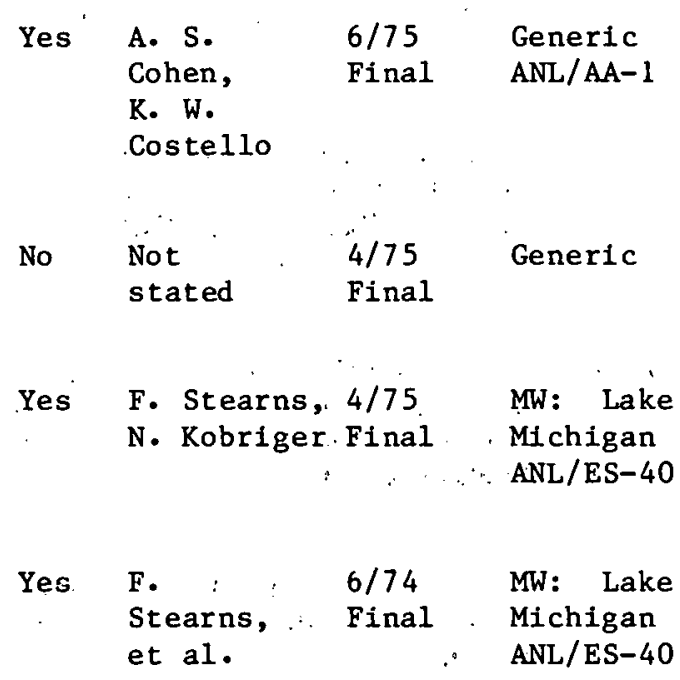

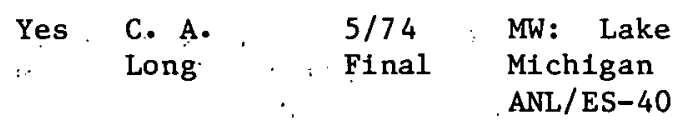

Yes R. B. $2 / 74$ MW: Lake Keener .. Final Michigan

ANL/ES-40 


\section{SUBJECT AREA: REGIONAL IMPACTS (Southeast)} ORN-MT-41 Annual Progress Report - Environ-
mental Sctiences Division

ORN-MT-13 Energy Conditions in the South: 1975

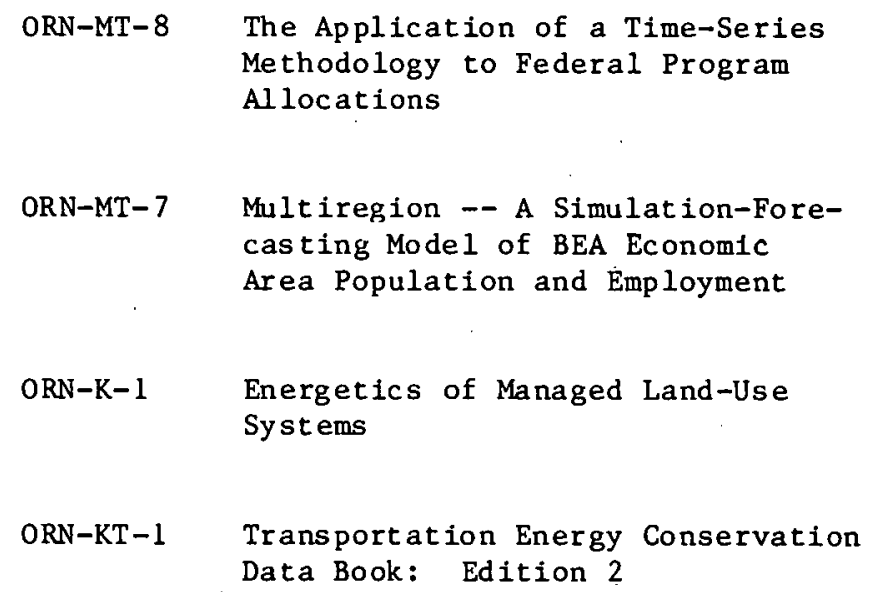

AS OF: APRIL 11, 1978

\begin{tabular}{|c|c|c|c|}
\hline Yes & $\begin{array}{l}\text { S. I. } \\
\text { Auerbach, } \\
\text { et al. }\end{array}$ & $\begin{array}{l}12 / 77 \\
\text { Final }\end{array}$ & $\begin{array}{l}\text { SE } \\
\text { ORNL-5257 }\end{array}$ \\
\hline Yes & $\begin{array}{l}\text { P. L. } \\
\text { Rice }\end{array}$ & $\begin{array}{l}12 / 77 \\
\text { Final }\end{array}$ & $\begin{array}{l}\mathrm{SE} \\
\text { ORNL/TM-556 }\end{array}$ \\
\hline Yes & $\begin{array}{l}\text { B. H. } \\
\text { Bronfman }\end{array}$ & $\begin{array}{l}11 / 77 \\
\text { Final }\end{array}$ & $\begin{array}{l}\text { Urban } \\
\text { ORNL/TM-611 }\end{array}$ \\
\hline Yes & $\begin{array}{l}\text { R. J. } \\
\text { olsen, } \\
\text { et al. }\end{array}$ & $\begin{array}{l}10 / 77 \\
\text { Final }\end{array}$ & $\begin{array}{l}\text { Genertc } \\
\text { ORNL/RUS-25 }\end{array}$ \\
\hline No & $\begin{array}{l}\text { J. M. } \\
\text { Klopatek }\end{array}$ & $\begin{array}{l}10 / 77 \\
\text { Final }\end{array}$ & Generic \\
\hline Yes & $\begin{array}{l}\text { D. B. } \\
\text { Shonka, } \\
\text { et al. }\end{array}$ & $\begin{array}{l}10 / 77 \\
\text { Final }\end{array}$ & $\begin{array}{l}\text { Generic } \\
\text { ORNL-5320 }\end{array}$ \\
\hline
\end{tabular}

No T. Wilbanks $10 / 77$ Generic

Draft ORNL-5351
Yes H. H. $\quad 9 / 77 \quad$ U. S. A. Shugart, Final ORNL/TM- et al. $\quad 6009$

\begin{tabular}{|c|c|c|c|}
\hline Yes & $\begin{array}{l}\text { B. J. } \\
\text { Purdy, } \\
\text { et al. }\end{array}$ & $\begin{array}{l}9 / 77 \\
\text { Final }\end{array}$ & $\begin{array}{l}\mathrm{NE} \\
\text { ORNL/NUREG/ } \\
\text { TM-22 }\end{array}$ \\
\hline Yes & $\begin{array}{l}\text { H. W. } \\
\text { Herzog, } \\
\text { R. J. } \\
\text { olsen }\end{array}$ & $\begin{array}{l}8 / 77 \\
\text { Final }\end{array}$ & $\begin{array}{l}\text { Generic } \\
\text { ORNL/TM-5918 }\end{array}$ \\
\hline
\end{tabular}




\section{SUBJECT AREA: REGIONAL IMPACTS (Sout heas $t$ )}

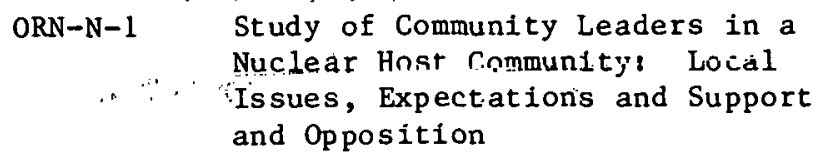

ORN-MT-6 Regional Studies Program Annual Report. :

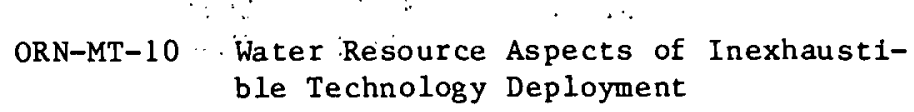

ORN-N-6 Citizens' Views About the Proposed Hartsville Nuclear Power Plant: A Survey of Resident's Perceptions in August 1975

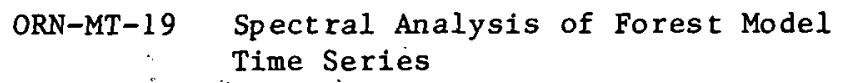

ORN-MT-I1 TEHM: A Terrestrial Ecosystem Hydrology Mode 1

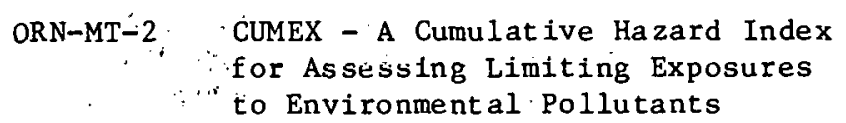

AS OF: APRIL 11,1978

$\begin{array}{llll}\text { Yes } & \text { B. H. } & 8 / 77 & \text { Tennesseo } \\ & \text { Bronfman } & \text { Final } & \text { ORNL/TM-5997 }\end{array}$

$\begin{array}{llll}\text { Yes } & \begin{array}{l}\text { R. M. } \\ \text { Davis, } \\ \text { et al. }\end{array} & \begin{array}{l}8 / 77 \\ \text { Final }\end{array} & \begin{array}{l}\text { SE } \\ \text { ORNL/TM-5838 }\end{array} \\ \text { No } & \begin{array}{l}\text { J. R. } \\ \text { Hyndman, } \\ \text { J. F. } \\ \text { Roach }\end{array} & \text { Final } & \text { Generic } \\ & & \\ \text { Yes } & \begin{array}{l}\text { E. D. } \\ \text { Sundstrom, Final } \\ \text { et al. }\end{array} & \text { ORNL/TM-5801 } \\ & & \end{array}$

$\begin{array}{llll}\text { No } & \text { W. R. } & 4 / 77 & \text { SE } \\ \text { Emanuel, } & \text { Final } & \text { ORNL-1059 } \\ \text { et al. } & & \end{array}$

$\begin{array}{clll}\text { Yes } & \begin{array}{l}\text { D. D. } \\ \text { Huff, } \\ \text { et al. }\end{array} & \begin{array}{l}4 / 77 \\ \text { Final }\end{array} & \begin{array}{l}\text { Generic } \\ \text { ORNL/NSF/ } \\ \text { EATC-27 }\end{array} \\ & & & \\ \text { Yes } & \begin{array}{l}\text { P. J. } \\ \text { Walsh, } \\ \text { et al. }\end{array} & \begin{array}{l}4 / 77 . \\ \text { Final }\end{array} & \begin{array}{l}\text { Gener1c } \\ \text { ORNL-5263 }\end{array} \\ & & \end{array}$

$\begin{array}{rlll}\text { Yes } & \begin{array}{l}\text { D. L. } \\ \text { Mielke, } \\ \text { et al. }\end{array} & \begin{array}{l}4 / 77 \\ \text { Final }\end{array} & \begin{array}{l}\text { Arkansas } \\ \text { ORNL/TM-5767 }\end{array} \\ \text { Yes } & \begin{array}{l}\text { R. E. } \\ \text { Moore }\end{array} & \begin{array}{l}4 / 77 \\ \text { Final }\end{array} & \begin{array}{l}\text { Generic } \\ \text { ORNL-5245 }\end{array}\end{array}$


SUBJECT AREA: REGIONAL IMPACTS (Southeast)

ORN-MT-12, Discrete Event Approach to Pollution

Transport and Vegetation Effects

ORN-MT-23 A Composite Hazard Index for Assessing Limiting Exposures to Environmental Pollutants -- Case Study

ORN-MT-18 Regional Analysis and Environmental Impact Assessment

ORN-MT-20 Spectral Analysis and Forest Dynamics: The Effects of Perturbations on Long-Term Dynamics

ORN-MT-21 RETADD: A Regional Trajectory and Diffusion-Deposition Model

ORN-MT-28 The Influence of Ground Cover on the Dry Disposition Rates of Gaseous Materials

ORN-MT-30 Regional Ecological Studies

ORN-MT-29 Structural Niches in a Forest Floor Small Mammal Community

ORN-MT-16 Development of an Appalachian Deciduous Forest Succession Model and its Application to Assessment of the Impact of the Chestnut Blight
AS OF: APRIL 11, 1978

$\begin{array}{llll}\text { Yes } & \begin{array}{l}\text { B. D. } \\ \text { Murphy, }\end{array} & \begin{array}{l}3 / 77 \ldots \\ \text { Kinal } .\end{array} & \begin{array}{l}\text { Generic } \\ \text { ORNL/CSD } \\ \text { Dixon }\end{array} \\ \text { No } & & \\ & \begin{array}{l}\text { E. M. } \\ \text { Rupp, } \\ \text { et al. }\end{array} & \begin{array}{l}1977 \\ \text { Draft }\end{array} & \text { Generic } \\ & & \end{array}$

$\begin{array}{lll}\text { No } & \text { D. C. } & \text { Not } \\ \text { Parzyck, } & \text { dated } \\ \text { et al. } & \text { Draft }\end{array}$

No

W. R.

1977

Emanuel, Final

SE et al.

No

C. L. Begovich,

1977

Draft

Generic et al:

Yes

B. D.
Murphy

$8 / 76$

Generic

Final

UCCND/

CSD-19

Yes

M. M. McCarthy et al.

$7 / 76$

SE

ORNL/RUS-24

Yes

R. D. Dheser,

$6 / 76$

Generic et al.

Fina 1 ORNL/TM-5004

No

H. $\mathrm{H}$. Shugart $5 / 76$ D. C. Final West 
SUBJECT AREA: REGIONAL IMPACTS (Southeas $t$ )

ORN-MT-27. Studies on Niche Theory and on
Ecological Successton at the
EDE/IBP Oak Ridge Site

\section{AS OF: APRIL 11, 1978}

No H. H. Not Generic

Shugart, Dated

et al. (At

least

1975)

Draft 
SUBJECT AREA: REGIONAL IMPACTS (Rocky Mounta1n)

LAS-MT-1 Breaking the Stalemate: An Analysis . of Boom Town Mitigation Policles

LAS-MT-2 Air Quality and Energy Development in the Rocky Mountain West

LAS-MT-3 Effects of Energy Development on Air Quality in the Rocky Mountain West

LAS-MT-13 A Time Series Profile of Urban Infrastructure Stocks in Selected Boom Towns in the Rocky Mountain States

LAS-MT-4 Air Optimization Model of Energy Related Economic Development in the Upper Colorado River Basin under Conditions of Water and Energy Resource Scarcity

LAS-MT-5 Methodology for the Analysis of the Impacts of Electric Power Production in the West: Executive Summary

LAS-CA-2 A Local Impact Model

LAS-MT-12 A Prototype for a Regional Energy Information System

Appropriate Technology and Navajo Economic Development
AS OF: "APRIL 11,1978

$\therefore \quad \therefore \quad \therefore \quad \therefore \quad \therefore . \therefore$

No

A. Ford $11 / 7.7$ Generic

Final LA-7046-MS

$\begin{array}{llll}\text { Yes } & \text { G. Hinman, } & 9 / 77 & \text { RM } \\ & \text { E. Leonard } & \text { Final } & \text { LA-6674 } \\ & & & \\ \text { No } & \text { G. Hinman, } 4 / 77 & \text { RM } \\ & \text { E. Leonard } & \text { Final } & \text { LA-UR-77- } \\ & & & 1057\end{array}$

Yes A. F. 4/77 RM

Mehr, Draft LA-6687-MS

R.

Cummings

Yes

G. E.

3/77 RM

Morris Final LA-6732-T

Yes

A. Ford, 1/77 W

H. W. Final LA-6720-PR

Lorber

Yes

M. D.

$1 / 77$

Generic

McKay, Final LA-6665-MS

L. A.

Bruckner

No

E. M.

$11 / 76$

Generic

Leonard, Final

LA-UR-76-1428

L. L.

Lynch

Yes

C. D.

$10 / 76$

RM

Kolsted, Final

LA-6489 
SUBJECT AREA: REGIONAL IMPACTS (Rocky Mountain)

LAS-MT-11 Proceedings Workshop on ERDA-Regional Program for the Rocky Mountain States
AS OF: APRIL 11,1978

No

$\begin{array}{lll}\text { R. E. } & 10 / 76 & \mathrm{RM} \\ \text { Malenfunt } & \text { Final } & \text { LA-6541-C }\end{array}$


SUBJECT AREA: REGIONAL IMPACTS (Far West)

$\begin{array}{cl}\text { LBL-MT-3 } & \begin{array}{l}\text { Potential Impacts of a } 1978 \text { California } \\ \text { Drought }\end{array} \\ \text { LBL-MT-4 } & \begin{array}{l}\text { A Water Conservation Scenario for } \\ \text { Residential and Industrial Sectors in } \\ \text { California: Potential Savings of Wate } \\ \text { and Related Energy }\end{array} \\ \text { LLL-MT-1 } & \begin{array}{l}\text { Pumping Energy Requirements for } \\ \text { Irrigation In California }\end{array} \\ \text { LBL-C-2 } & \begin{array}{l}\text { Impacts of Future Coal Use in } \\ \text { California - Interim Report, NCUA }\end{array} \\ \text { LAS-MT-14 } & \text { Energy Flow Patterns for } 1975\end{array}$

LBL-MT-1 Effects of the Drought on California Electricity Supply and Demand

Work Statement for an Assessment of Coal Utilization in California

LBL-MT-2 Production Cycles in Aquatic Microcosms

LLL-N-5 A Comparison of In Situ Gamma Soil Analysis and Soil Sampling Data for Mapping $241_{\mathrm{Am}}$ and $239_{\mathrm{Pu}}$ Soil Concentrations at the Nevada Test Site
AS OF: APRIL 11, 1978

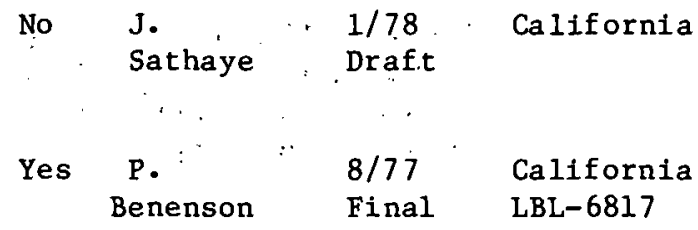

$\begin{array}{lll}\text { No G. T. } & 7 / 77 . & \text { California } \\ \text { Knutson, } & \text { Final } & \text { Spec. Pub. } \\ \text { et al. } & & 3215\end{array}$

No W. Siri, $7 / 77$ California et al. Final UCID-3941

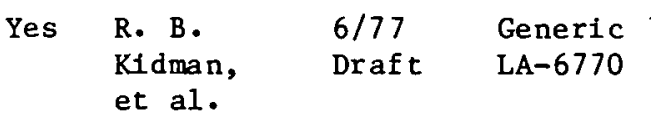

\begin{tabular}{|c|c|c|c|}
\hline No & $\begin{array}{l}\text { P. } \\
\text { Benenson, }\end{array}$ & $\begin{array}{l}6 / 77 \\
\text { Final }\end{array}$ & $\begin{array}{l}\text { California } \\
\text { LBL-6356 }\end{array}$ \\
\hline
\end{tabular}

No Not stated $\begin{array}{ll}3 / 77 & \text { Final }\end{array} \quad$ California

$\begin{array}{llll}\text { Yes } & \text { A. } & 2 / 77 & \text { Generic } \\ & \text { Jassby, } & \text { Final } & \text { LBL-5965 } \\ \text { et al. } & & \end{array}$

$\begin{array}{llll}\text { No J. Kirby, } & 11 / 76 & \text { Nevada } \\ \text { et al. } & \text { Draft } & \text { UCRL-78273 }\end{array}$


SUBJECT AREA: REGIONAL IMPACTS (Far West)

LLL-MT-4

The 1972 Flow of Electrical Energy

In California and its Relationship

to Selected County Level Charac-

teristic Parameters

LAS-MT-8 Summary Description of the BOOM 1 Mode 1

LAS-MT-9 User's Gulde to the BOOM 1 Model
AS OF: APRIL 11, 1978

Yes

K. Haven $10 / 76$

Final

Calif ornia

UCRL-52143

Yes

A. Ford

$9 / 76$

Final

Generic

LA-6424-MS

Yes

A. Ford

$8 / 76$

Final

Generic

LA-6396-MS 
SUBJECT AREA: REGIONAL IMPACTS (Pacific Northwest)

PNL-MT-1 Energy and Water Consumption of Pacific Northwest Irrigation Systems

PNL-MT-5 The ELSA/ELDA Model, Linking Methodology and Simulation Results $\begin{array}{ll}\text { PNL-MT-2 } & \text { Organizations Influencing Northwest } \\ \text { Energy Policy and Management }\end{array}$

PNL-C-6 Big Game Resource in the Powder River Basin Region, Montana-Wyoming

PNL-CC-2 Preliminary Assessment of the Health and Environmental Effects of Coal Utilization in the Pacific Northwest

PNL-MT-6 Social Impact Assessment and Management Methodology Using Soclal Indicators and P1.anning Strategies

PNL-CC-3 The Management of Social and Economic Impacts As sociated with the Construction of Large-Scale Projects: Experience from the Western Coal Development Communities.

PNL-MT-3 Energy Policy and Public Acceptance: Current and Future Directions

PNL-N-1 Citizen Involvement in Energy Decision Making
- AS OF: APRIL $11,1978$.

Yes

L. King, $9 / 77$, NW

et al. Final BNlL-RAP-19

No

A. E. - Davis,

$9 / 77$

NW

D. W.

Draf $t$

PNL-RAP- 17

Fraley

Yes

G. L.

Wilfert

$8 / 77$

M

Final BNWL-RAP-20

UC -11

Yes

W. H.

Rickard

$7 / 77$

NW

Final

BNWL-2084RAP- 11

No

J. Burnham

$7 / 77$

NW

Draft PNL-RAP-21

No

M. E.

Olsen,

$7 / 77$

Generic

et al.

Draft

BNWL-20-84, RAP - 18

Yes
M. Greene,
M. Curry
$6 / 77$
NW
Final
BNWL-RAP- 16

Yes

M. Curry,

$5 / 77$

NW et al.

Final

BNWL-2084RAP -15

Yes

M. Curry,
M. Olsen

$3 / 77$

NW

Final

BNWL-2084RAP- 14 
SUBJECT AREA: REGIONAL IMPACTS (Pacific Northwest)

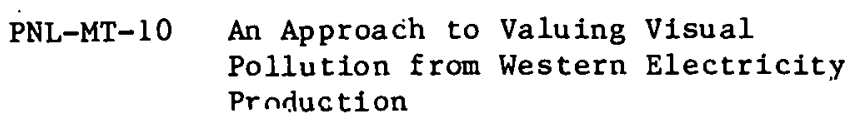

PNL-MT-12 Power Plant Construction: Productivity and Construction Period

PNL-MT-13 Social, Values and Environmental Assessment in Thermal Power Plant Siting

PNL-MT-4 ELSA: An Electric Power Supply Analysis Model for the Pacific Northwest

PNL-K-1 Conserving Energy by Changing Societal Goals

PNL-CC-4

Estimating Agricultural Production and its Response to $\mathrm{SO}_{2}$ in the Pacific Northwest

PNL-MT-7 Systems Methodology for Assessing the Demographic Implications of Energy Development

PNL-MT-8 Toward a Methodology for Conducting Social Impact Assessments Using Quality of Soclal Life Indicators

$$
\text { PNL-C-5 }
$$

A Program Plan for Performing Soctal Impact Assessment: A Case Study of Coal Development in the Powder River Region

PNL-MT-I1 Pacific Northwest Regional Assessment Program 1975 Annual Report

\section{AS OF : . APRIL 11,1978}

$\begin{array}{llll}\text { Yes } & \text { L. E. } & 2 / 77 \\ & \text { Ericksull } & \text { Final }\end{array}$. BNWL-2103

Yes S. C. . 2/77 Genertc Schute Final BNWL-2124

$\begin{array}{llll}\text { No J. C. } & 2 / 77 & \text { Generic } \\ & \text { King } & \text { Final } & \text { BNWL-SA-6065 }\end{array}$

Yes T. P. $11 / 76 \quad \mathrm{NW}$

Harrington, Final BNWL-2084J. J. RAP- 8 Jacobsen

No M. E. $10 / 76$ Generic Olsen. Final

Yes

R. Sauer

$10 / 76$

NW

Final BNWL-2084RAP-10

$\begin{array}{llcl}\text { Yes } & \begin{array}{l}\text { J. J. } \\ \text { Jacobsen }\end{array} & \begin{array}{l}9 / 76 \\ \text { Final }\end{array} & \begin{array}{l}\text { Generic } \\ \text { BNWL-2084- } \\ \text { RAP-6 }\end{array} \\ \text { Yes } & \begin{array}{l}\text { M. E. } \\ \text { Olsen, } \\ \text { et al. }\end{array} & \begin{array}{l}7 / 76, \\ \text { Final }\end{array} & \begin{array}{l}\text { Generic } \\ \text { BNWL-2084- } \\ \text { RAP-2 }\end{array} \\ \text { Yes } & \begin{array}{l}\text { M. Curry, } \\ \text { et al. }\end{array} & \begin{array}{l}\text { 6/76 } \\ \text { Final }\end{array} & \begin{array}{l}\text { NW } \\ \text { BNWL-2084- } \\ \text { RAP-1 }\end{array}\end{array}$

$\begin{array}{lll}\text { Yes Not stated } & 6 / 76 & \text { NW } \\ & \text { Final } & \text { BNWL-2084- } \\ & & \text { RAP-1 }\end{array}$


SUBJECT AREA: REGIONAL IMPACTS (Pacif ic Northwest)

PNL-C-7 A New Look at Low Sulfur Coal Resources
in Montana and Wyoming

PNL-MT-9

Environmental and Socioeconomic Aspects of Energy in the Pacific Northwest
AS OF: APRIL 11, 1978

No

D. S.

$6 / 76$

NW

Renine Final BNWL-SA-5847

No

H. Hollis- $12 / 75$ NW

ter, Final

W. H.

Swift 


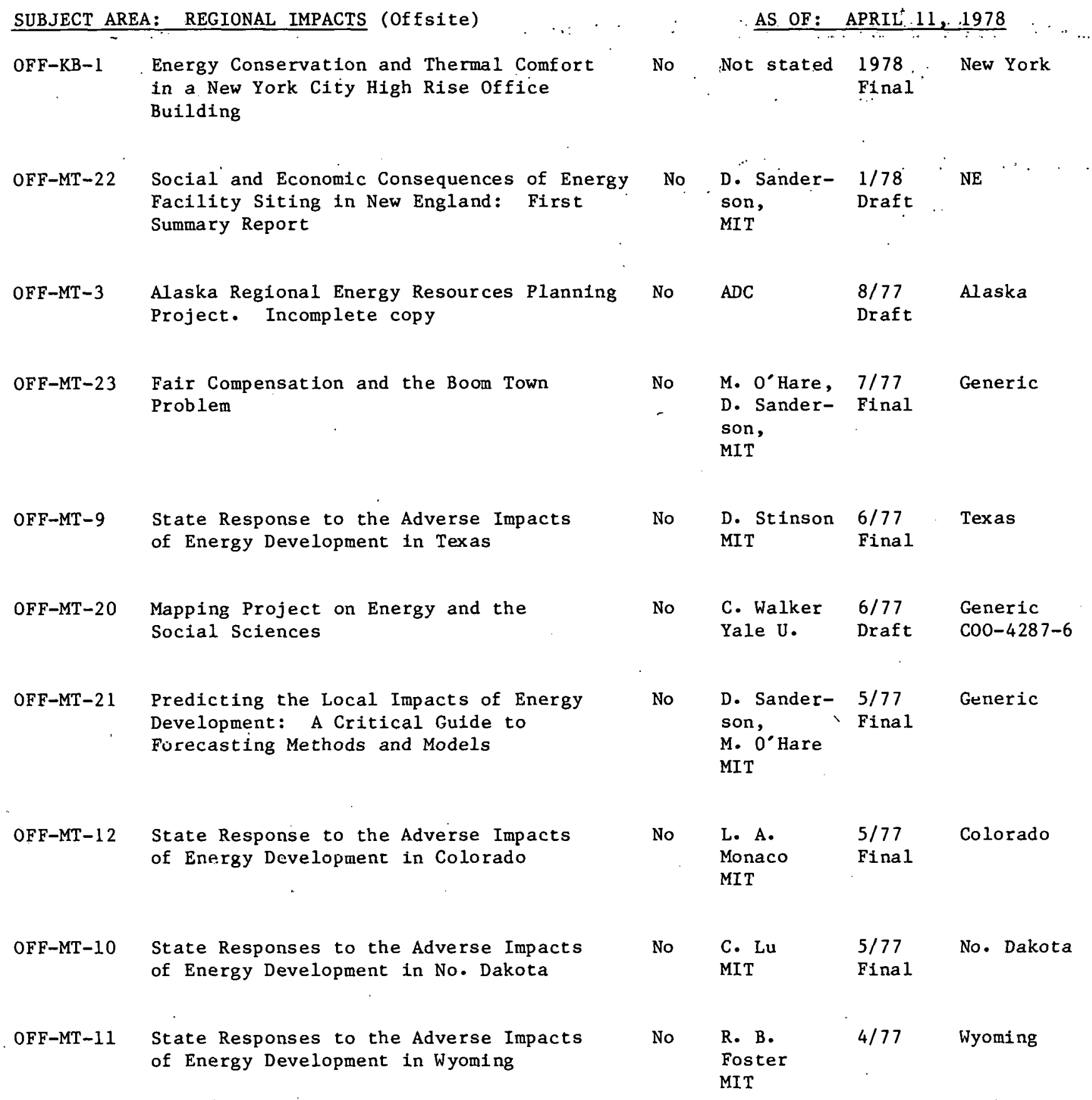


SUBJECT AREA: REGIONAL IMPACTS (Offsite)

OFF-MT-19 EIA Review
AS OF: APRIL 11,1978

No

Not Genertc

Lairdman stated

MIT Final 
